\title{
Topology Optimization of Geometrically Nonlinear Trusses with Spurious Eigenmodes Control
}

\author{
Lei $\mathrm{Li}^{1}$ and Kapil Khandelwal ${ }^{2}$
}

\begin{abstract}
In this paper, topology optimization of geometrically nonlinear trusses with and without stability constraints is investigated. It is shown that if classical minimum compliance formulation is considered without any stability constraints, the optimized designs are unstable and convergence issues may be encountered in the nonlinear structural analyses. To address these issues, a minimum compliance formulation with critical load factor constraint is proposed together with a strategy based on spurious modal energy ratio to determine the true critical eigenmodes and the corresponding critical load factor. Several numerical examples are presented to demonstrate the effectiveness of the proposed approach, which show that the optimized truss topologies obtained using the proposed approach are stable. More importantly, the critical load constraint is able to guarantee that the first critical load of the optimized design is always above the applied load so that the proposed approach is free from convergence issues during the Newton Raphson solution process.
\end{abstract}

Keywords: Truss topology optimization; Geometric nonlinearity; Critical load constraint; Spurious Eigen-modes; Nonlinear systems. 
2 Topology optimization of trusses is an important topic in structural optimization as it provides an

3 efficient and flexible design technique [1-5]. Unlike classic sizing or shape optimization,

4 topology optimization seeks the best layout of members by optimizing the given objective

5 function while satisfying the prescribed constraints and boundary conditions [6, 7]. The truss

6 topology optimization problem is usually formulated and implemented in the framework of a

7 ground structure approach [8]. In this approach, an interconnected initial mesh, termed "ground

8 structure", is first generated, and then the inefficient members are subsequently removed during

9 the optimization process. Small deformation and elastic material assumption is usually made in

10 the truss topology optimization, which results in a geometrically linear truss model. In this case,

11 the most commonly used optimization formulation is minimum compliance, subjected to the

12 equilibrium equations and volume constraints, which is also called the stiffness design

13 formulation [9-12]. This displacement based non-convex formulation can be either solved by

14 various nonlinear programming (NLP) methods [7, 13], or transformed into convex formulations

15 which are solved by tailored optimization algorithms [12]. The stiffness design formulation can

16 be also approximated in terms of member forces to yield a linear programming (LP) problem,

17 which can be solved very efficiently with a large number of design variables [14-16].

18 As stability considerations are absent from the stiffness design formulations, the optimized 19 topologies may be unstable. For topology optimization, a number of studies in the past have 20 addressed the local and global stability issues in linear elastic truss topology optimization [17].

21 In most cases, the local stability of members is enforced by including constraints based on Euler

22 buckling [18-20]. While the local stability issue can be addressed in the stiffness design 23 formulations by including additional constraints based on the Euler buckling criterion, the final 
1 optimized topologies can still be globally unstable, as in the common case of a chain of

2 collinearly connected truss members [18]. Although these collinear members can be merged into

3 a one longer member through the node cancellation post-processing, it is shown that the long

4 members also increase the potential for local instabilities, and the global stability may still not be

5 ensured [19]. To address this issue, linear truss topology optimization considering global stability

6 has been investigated in the past. For instance, Ben-Tal et al. [17] and Kočvara [21] included a

7 linear global stability constraint into the compliance formulation. Guo et al. [22] incorporated

8 overlapping bars in the ground structure aiming to address the difficulty caused by hinge

9 cancellation pointed out by Rozvany [18].

10 These aforementioned studies are mostly based on stiffness design formulation and are solved

11 using NLP, since the LP formulations cannot be recovered in the case where stability constraints

12 are included. Moreover, all the studies mentioned above are restricted to linear elastic truss

13 models, and the so-called linear buckling analysis is adopted to evaluate the critical loads. The

14 linear buckling analysis is based on the assumption that geometric nonlinearities are insignificant

15 and this assumption can lead to incorrect estimation of the critical loads if the actual

16 deformations are large [23]. Therefore, it is necessary to employ geometrically nonlinear models

17 in the stability analysis for accurately determining the critical loads. However, this will lead to a

18 nonlinear system, and the equilibrium solution of such a system has to be characterized with

19 respect to critical points including bifurcation and limit points. Such nonlinear analysis is also

20 rather meaningful, since it will naturally capture the nonlinear behavior of truss systems under

21 large deformations. Thus topology optimization of geometrically nonlinear trusses is the focus of

22 this paper. It should be noted that only the literature considering truss topology optimization is

23 reviewed above; details about nonlinear topology optimization in continuum settings can be 
1 found in Refs [24, 25] and references therein. In addition, sizing optimization of geometrical

2 nonlinear trusses is not reviewed here and the details can be found elsewhere [26-29].

3 For geometrically nonlinear trusses, Ramos and Paulino [30] recently proposed a convex

4 topology optimization formulation using a potential energy approach. However, stability issues

5 were not addressed in that study. In this study, topology optimization of geometrically nonlinear

6 trusses is carried out using optimization formulations with and without critical load constraints.

7 To this end, a new optimization formulation is proposed for nonlinear truss topology

8 optimization, wherein the global stability constraint is explicitly incorporated using the minimum

9 critical load associated with the true eigenmode. To address the spurious modes issue associated

10 with the low density members [31-33], a new spurious mode identification approach is presented

11 based on the spurious modal energy ratio. Various test cases are presented to demonstrate the

12 effectiveness of the proposed approaches for handling stability issues in geometrically nonlinear

13 trusses.

14 The paper is organized as follows: In Section 2, the large deformation kinematics/kinetics of 15 geometrically nonlinear truss, the equilibrium equations and solution techniques are presented.

16 Section 3 presents the critical load factor estimation based on the nonlinear analysis. In Section 4,

17 the topology optimization formulations are stated and the relevant sensitivity analyses are carried

18 out. Section 5 describes the approach to identify the localized eigenmodes using spurious modal 19 strain energy ratio. Various test cases are presented in Section 6 to demonstrate the effectiveness 20 of the proposed approaches. Finally, the important remarks and conclusions are given in Section 217.

\section{2. Geometrically Nonlinear Truss Analysis}


1 In this section, a geometrically nonlinear hyperelastic truss model based on Green-Lagrangian

2 strain is briefly described in which the large deformation kinematics are taken into consideration.

\section{$3 \quad 2.1$ Kinematics and kinetics}

4 Consider a truss member $A B$ in initial configuration with initial length $L_{0}$, area $A_{0}$ and volume

$5 V_{0}=L_{0} A_{0}$ as shown in Figure 1. After deformation $\boldsymbol{u}_{a}$ and $\boldsymbol{u}_{b}$ of nodes $A$ and $B$, respectively,

6 the current configuration for the member becomes $a b$ with member length $L$. The member length

7 before and after the deformation are given by

$$
\begin{aligned}
& L_{0}=\sqrt{\boldsymbol{X}_{B A}^{T} \boldsymbol{X}_{B A}} \\
& L=\sqrt{\left(\boldsymbol{X}_{B A}+\boldsymbol{u}_{b a}\right)^{T}\left(\boldsymbol{X}_{B A}+\boldsymbol{u}_{b a}\right)}
\end{aligned}
$$

8 where the coordinate difference vector $\boldsymbol{X}_{B A}$ and the relative displacement vector $\boldsymbol{u}_{b a}$ between

$9 \quad$ nodes $A$ and $B$ are given as

$$
\begin{aligned}
& \boldsymbol{X}_{B A}=\left[X_{B A}, Y_{B A}, Z_{B A}\right]^{T}=\left[X_{B}-X_{A}, Y_{B}-Y_{A}, Z_{B}-Z_{A}\right]^{T} \\
& \boldsymbol{u}_{b a}=\left[u_{b a}, v_{b a}, w_{b a}\right]^{T}=\left[u_{b}-u_{a}, v_{b}-v_{a}, w_{b}-w_{a}\right]^{T}
\end{aligned}
$$

10 where $\left(X_{A}, Y_{A}, Z_{A}\right)$ and $\left(X_{B}, Y_{B}, Z_{B}\right)$ are the nodal coordinates in the initial configuration of

11 member nodes $A$ and $B$, while $\left(u_{a}, v_{a}, w_{a}\right)$ and $\left(u_{b}, v_{b}, w_{b}\right)$ are the displacements of the nodes $A$ 12 and $B$, respectively, as shown in Figure 1. The Green-Lagrangian strain is used in this study

13 which is given by

$\varepsilon=\frac{1}{2}\left(\lambda^{2}-1\right)$

14 where $\lambda=L / L_{0}$ is the stretch ratio for the truss member. Note that the other strain measures such 15 as logarithmic strain, Euler-Almansi strain, etc. [34], can also be employed to formulate the finite 16 deformation truss model [30]. Only the elastic material behavior is considered and, in this case, 17 the stress is given by 
4 The internal $\left(W_{\text {int }}\right)$ and external $\left(W_{\text {ext }}\right)$ virtual work for the truss structure can be expressed as

$$
\begin{aligned}
& W_{\text {int }}=\sum_{e=1}^{n_{e l}} W_{i n t}^{e}=\delta \boldsymbol{u}^{T} \boldsymbol{F}_{\text {int }}(\boldsymbol{u}) \text { where } \\
& \boldsymbol{F}_{\text {int }}(\boldsymbol{u})=\underset{e=1}{n_{e l}} \boldsymbol{F}_{\text {int }}^{e}=\underset{e=1}{n_{e l}}\left(\boldsymbol{B}^{e^{T}} A_{0}^{e} L_{0}^{e} \sigma^{e}\right) \\
& W_{\text {ext }}=\delta \boldsymbol{u}^{T} \boldsymbol{P}
\end{aligned}
$$

5 where $\mathcal{A}$ is the standard finite element assembly operator, $n_{e l}$ denotes the total number of truss

6 members, $\boldsymbol{P}$ is the external nodal force vector and $\delta \boldsymbol{u}$ is the global virtual displacement vector.

7 Also, the displacement-gradient operator $\boldsymbol{B}^{e}$ for the $e^{\text {th }}$ member is given by

$\boldsymbol{B}^{e}=\frac{1}{L_{0}^{e^{2}}}\left[-X_{B A}^{e}-u_{b a}^{e},-Y_{B A}^{e}-v_{b a}^{e},-Z_{B A}^{e}-w_{b a}^{e}, X_{B A}^{e}+u_{b a}^{e}, Y_{B A}^{e}+v_{b a}^{e}, Z_{B A}^{e}+w_{b a}^{e}\right]$

8 The principle of virtual work implies that $W_{\text {int }}=W_{\text {ext }} \forall \delta \boldsymbol{u}$, which after the application of

9 boundary conditions yields the equilibrium equations in residual form as

$\boldsymbol{R}(\boldsymbol{u})=\boldsymbol{F}_{\text {int }}(\boldsymbol{u})-\boldsymbol{P}=\mathbf{0}$

10 The tangent stiffness matrix that is needed in the solution process is given by 
$\boldsymbol{K}_{T}(\boldsymbol{u})=\frac{\partial \boldsymbol{F}_{\text {int }}(\boldsymbol{u})}{\partial \boldsymbol{u}}=\boldsymbol{K}_{0}+\boldsymbol{K}_{u}(\boldsymbol{u})+\boldsymbol{K}_{\sigma}(\boldsymbol{u})$

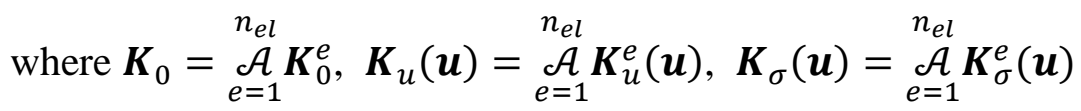

1 In Eq. (11), the tangent stiffness $\boldsymbol{K}_{T}(\boldsymbol{u})$ is divided into three parts where: $\boldsymbol{K}_{0}$ is the initial

2 stiffness component, $\boldsymbol{K}_{u}(\boldsymbol{u})$ is the displacement stiffness and $\boldsymbol{K}_{\sigma}(\boldsymbol{u})$ is the stress stiffness, which

3 are assembled by using their local counterparts, respectively. Using the chain rule of

4 differentiation, the tangent stiffness components for a truss member are given by

$\boldsymbol{K}_{0}^{e}=\frac{A_{0}^{e} E^{e}}{L_{0}^{e 3}}\left[\begin{array}{cc}\overline{\boldsymbol{K}_{0}^{e}} & -\overline{\boldsymbol{K}_{0}^{e}} \\ -\overline{\boldsymbol{K}_{0}^{e}} & \overline{\boldsymbol{K}_{0}^{e}}\end{array}\right], \boldsymbol{K}_{u}^{e}=\frac{A_{0}^{e} E^{e}}{L_{0}^{e 3}}\left[\begin{array}{cc}\overline{\boldsymbol{K}_{u}^{e}} & -\overline{\boldsymbol{K}_{u}^{e}} \\ -\overline{\boldsymbol{K}_{u}^{e}} & \overline{\boldsymbol{K}_{u}^{e}}\end{array}\right], \boldsymbol{K}_{\sigma}^{e}=\frac{A_{0}^{e} \sigma^{e}}{L_{0}^{e}}\left[\begin{array}{cc}\boldsymbol{I} & -\boldsymbol{I} \\ -\boldsymbol{I} & \boldsymbol{I}\end{array}\right]$

where

$\overline{\boldsymbol{K}_{0}^{e}}=\left[\begin{array}{ccc}X_{b a}^{e}{ }^{2} & X_{b a}^{e} Y_{b a}^{e} & X_{b a}^{e} Z_{b a}^{e} \\ X_{b a}^{e} Y_{b a}^{e} & Y_{b a}^{e} & Y_{b a}^{e} Z_{b a}^{e} \\ X_{b a}^{e} Z_{b a}^{e} & Y_{b a}^{e} Z_{b a}^{e} & Z_{b a}^{e}{ }^{2}\end{array}\right]$

$\overline{\boldsymbol{K}_{u}^{e}}=\left[\begin{array}{ccc}u_{b a}^{e}+2 u_{b a}^{e} X_{b a}^{e} & u_{b a}^{e} v_{b a}^{e}+v_{b a}^{e} X_{b a}^{e}+u_{b a}^{e} Y_{b a}^{e} & u_{b a}^{e} w_{b a}^{e}+w_{b a}^{e} X_{b a}^{e}+u_{b a}^{e} Z_{b a}^{e} \\ u_{b a}^{e} v_{b a}^{e}+v_{b a}^{e} X_{b a}^{e}+u_{b a}^{e} Y_{b a}^{e} & v_{b a}^{e}+2 v_{b a}^{e} Y_{b a}^{e} & v_{b a}^{e} w_{b a}^{e}+w_{b a}^{e} Y_{b a}^{e}+v_{b a}^{e} Z_{b a}^{e} \\ u_{b a}^{e} w_{b a}^{e}+w_{b a}^{e} X_{b a}^{e}+u_{b a}^{e} Z_{b a}^{e} & v_{b a}^{e} w_{b a}^{e}+w_{b a}^{e} Y_{b a}^{e}+v_{b a}^{e} Z_{b a}^{e} & w_{b a}^{e}+2 w_{b a}^{e} Z_{b a}^{e}\end{array}\right]$

5 and $\boldsymbol{I}$ is the $3 \times 3$ identity matrix. It is important to note that $\boldsymbol{K}_{0}$ is independent of the

6 displacement $\boldsymbol{u}$, while $\boldsymbol{K}_{u}(\boldsymbol{u})$ and $\boldsymbol{K}_{\sigma}(\boldsymbol{u})$ depend on displacement, which introduce the

7 geometrical nonlinearity into the system. In addition, if only $\boldsymbol{K}_{0}$ is considered, the nonlinear truss

8 model degenerates to the geometrically linear case.

\section{$9 \quad 2.3$ Solution using load control method}

10 To solve the nonlinear problem (Eq. (10)) using the load control method, the total external load

$11 \boldsymbol{P}$ is incrementally applied within an iterative Newton-Raphson (NR) scheme [35]. In this study,

12 the load is applied in $N$ equal increments such that at the $n^{\text {th }}$ step the applied load is $\gamma_{n} \boldsymbol{P}$, where

$13 \gamma_{n}=n / N$ is the prescribed load factor at step $n(n=0,1, \ldots N)$. Note that the initial conditions 
1 are specified such that at $\gamma^{0}=0$ and the corresponding displacement vector is $\boldsymbol{u}_{0}=\mathbf{0}$. The

2 equilibrium equations at the $n^{\text {th }}$ load step are given by

$$
\boldsymbol{R}\left(\boldsymbol{u}_{n}^{k}\right)=\boldsymbol{F}_{\text {int }}\left(\boldsymbol{u}_{n}^{k}\right)-\gamma_{n} \boldsymbol{P}=\mathbf{0}
$$

3 Equation (15) is solved using the NR process, and the predictor to start the NR process is given

4 by $\boldsymbol{u}_{n}^{0}=\boldsymbol{u}_{n-1}$, where $\boldsymbol{u}_{n-1}$ is the displacement vector for the last converged step and $\boldsymbol{u}_{n}^{k}$

$5 \quad(k=0,1,2, \ldots)$ is the iterative displacement at the $n^{t h}$ step. The correctors, $\Delta \boldsymbol{u}_{n}^{k}$, at a NR

6 iteration step are obtained by Eq. (16) as

$\Delta \boldsymbol{u}_{n}^{k}=-\left[\boldsymbol{K}_{T}\left(\boldsymbol{u}_{n}^{k}\right)\right]^{-1} \boldsymbol{R}\left(\boldsymbol{u}_{n}^{k}, \gamma_{n}\right)$

$\boldsymbol{u}_{n}^{k+1}=\boldsymbol{u}_{n}^{k}+\Delta \boldsymbol{u}_{n}^{k}$

7 where $\boldsymbol{K}_{T}\left(\boldsymbol{u}_{n}^{k}\right)$ is the tangent stiffness matrix evaluated at $\boldsymbol{u}_{n}^{k}$, and finally the displacements are

8 updated using Eq. (17). The process is repeated until the convergence is achieved.

\subsection{Arc-length method and branch switching technique}

The load control method may fail when critical points - such as bifurcation or limit points - are encountered on the solution path [36]. In this case, it might not be possible to find the solution of the nonlinear problem for the applied load $\boldsymbol{P}$ in Eq. (10). At critical points, the tangent stiffness matrix $\boldsymbol{K}_{T}$ becomes singular, and the NR process (Eq. (16)) cannot be applied. To handle the limit points, more sophisticated path-following solution approaches such as the arc-length methods are used $[37,38]$. Unlike the load control method, in addition to the displacement $\boldsymbol{u}_{n}$ at $n^{\text {th }}$ step, the load factor $\gamma_{n}$ also becomes a variable in the arc-length method. The equilibrium equation Eq. (15) is then expressed as

$$
\boldsymbol{R}\left(\boldsymbol{u}_{n}^{k}, \gamma_{n}\right)=\boldsymbol{F}_{\text {int }}\left(\boldsymbol{u}_{n}^{k}\right)-\gamma_{n} \boldsymbol{P}=\mathbf{0}
$$

and an additional arc-length constraint is introduced. In this study, a cylindrical arc-length constraint is used [38], and this constraint is given by 
$\psi\left(\boldsymbol{u}_{n}^{k}, \gamma_{n}\right)=\left(\boldsymbol{u}_{n}^{k}-\boldsymbol{u}_{n-1}\right)^{T}\left(\boldsymbol{u}_{n}^{k}-\boldsymbol{u}_{n-1}\right)-\Delta s^{2}=0$

1 where $\boldsymbol{u}_{n}^{k}$ is the iterative displacement at $n^{\text {th }}$ step and $\Delta s$ is the prescribed arc-length increment.

2 Equations (18) and (19) are simultaneously solved for the knowns $\boldsymbol{u}_{n}^{k}$ and $\gamma_{n}$ using the NR

3 method and further details can be found in Ref. [39].

4 The arc-length method can trace the primary solution path and can handle critical points that 5 correspond to the limit points. However, when a critical point is the bifurcation point, there are

6 more than one solution branches, and the treatment of bifurcation including the calculation of 7 solution branches requires additional considerations [36]. To handle both the limit and

8 bifurcation points, a critical point along the solution path is first computed using the condition 9 that $\operatorname{det}\left[\boldsymbol{K}_{T}\right]=0$ at critical points. In this study, the minimum eigenvalue $\lambda_{\min }$ of $\boldsymbol{K}_{T}$ is used to track the critical points and a bisection method is used to find the exact critical point $\left(\boldsymbol{u}_{c}, \gamma_{c}\right)$

11 where $\lambda_{\min }=0$ [36]. Only simple critical points are considered in this study, implying that only

12 one eigenvalue is zero and there are (at most) two solution branches at a bifurcation point. The 13 eigenvector, $\boldsymbol{\phi}_{c}$, corresponding to the zero eigenvalue is determined, and the nature of critical 14 point is then established using the following criteria [40]

$\boldsymbol{\phi}_{c}^{T} \boldsymbol{P}=0 \Rightarrow$ Bifurcation point

$\boldsymbol{\phi}_{c}^{T} \boldsymbol{P} \neq 0 \Rightarrow \quad$ Limit point

15 At a bifurcation point, the eigenvector $\boldsymbol{\phi}_{c}$ gives the direction of the secondary solution path, and this eigenvector is used for tracing the secondary solution path. The perturbation for determining

17 the secondary solution path is performed by adding the scaled eigenvector to the deformed 18 configuration at critical points $\left(\boldsymbol{u}_{c}\right)$ as

$\boldsymbol{u}_{b}=\boldsymbol{u}_{c}+\xi_{0} \frac{\boldsymbol{\phi}_{c}}{\left\|\boldsymbol{\phi}_{c}\right\|}$ 
1 where $\boldsymbol{u}_{b}$ is the perturbed displacement on the secondary branch and $\xi_{0}$ is the perturbation

2 magnitude that is estimated by

$\xi_{0}=\frac{\left\|\boldsymbol{u}_{c}\right\|}{\tau_{0}}$

3 where $\tau_{0} \approx 100$ and should be adjusted until the perturbation is able to find the secondary

4 solution branch [36]. The arc-length and secondary branch switching method described above is

5 implemented within the framework of nonlinear finite element analysis mentioned in Section 2.2.

6 The detailed algorithm for the calculation of equilibrium branches is provided in Table 1.

\begin{tabular}{|c|c|}
\hline Step & Algorithm \\
\hline (1) & $\begin{array}{l}\text { Calculate the equilibrium state using arc-length method Eq. (18) and } \\
\text { Eq. (19). }\end{array}$ \\
\hline (2) & Track the minimal eigenvalue $\lambda_{\min }$ of the tangent stiffness matrix $\boldsymbol{K}_{T}$. \\
\hline (3) & $\begin{array}{l}\text { If negative } \lambda_{\min } \text { occurs: critical point has been passed. Bracket the } \\
\text { critical point using last step information. }\end{array}$ \\
\hline (4) & Use bisection method to determine the critical point. \\
\hline (5) & If the critical point is a limit point, go to (1) \\
\hline (6) & $\begin{array}{l}\text { Else-if: the critical point is bifurcation point. Use branch switching } \\
\text { algorithm. }\end{array}$ \\
\hline (6.1) & If primary branch is still needed, go to (1). \\
\hline$(6.2)$ & Find the eigenvector, $\boldsymbol{\phi}_{c}$, at the critical point. \\
\hline (6.3) & $\begin{array}{l}\text { Add perturbation to the critical displacement } \boldsymbol{u}_{c} \text { using Eq. (21) } \\
\text { and Eq. (22). }\end{array}$ \\
\hline (6.4) & If secondary branch has been found, go to (7). \\
\hline (6.5) & Else, change the value of $\xi_{0}$ and go to (6.3). \\
\hline (7) & $\begin{array}{l}\text { Continue to calculate the equilibrium state using arc-length method Eq. } \\
\text { (18) and Eq. (19). }\end{array}$ \\
\hline (8) & If other secondary path needs to be calculated, go to (6.3). \\
\hline (9) & Finish if all needed branches are calculated. \\
\hline
\end{tabular}

\section{3. Critical Load Factor Analysis}

9 Structural stability is an important aspect of structural safety and design, and requires special 10 consideration in the topology optimization process. An equilibrium point $(\boldsymbol{u}, \gamma)$ is considered to 
1 be stable if the tangent stiffness matrix $\boldsymbol{K}_{T}$ is positive definite at $(\boldsymbol{u}, \gamma)$, and a solution branch is

2 stable if $\boldsymbol{K}_{T}$ is positive definite at all the points on that branch. From a mathematical viewpoint,

3 the transition from stability to instability can only occur at critical points, which are related to a

4 critical equilibrium state, $\left(\boldsymbol{u}_{c}, \gamma_{c}\right)$, where the tangent stiffness matrix $\boldsymbol{K}_{T}$ becomes singular. If a

5 critical point is the limit point, the load on the structure cannot be further increased and the

6 stability is lost. On the other hand, if a critical point is a bifurcation point, it might be possible to

7 find a solution branch on which the load can be increased; however, this solution branch may

8 still be unstable. Therefore, in most of the cases, the critical load, $\boldsymbol{P}_{c} \stackrel{\text { def }}{=} \gamma_{c} \boldsymbol{P}$, associated with the

9 critical point is the maximum load that can be safely applied to the system. The direct

10 identification of critical points using arc-length and bisection method is described in Section 2.4.

11 However, this direct method requires large computational effort, and is not suitable for topology

12 optimization where the critical point analysis has to be repeated at each design iteration.

13 The critical load factor can also be approximated to circumvent the associated computational

14 cost. However, the quality of results will depend on the approximations used in the critical point

15 analysis. Let $\boldsymbol{K}_{T}\left(\boldsymbol{u}_{c}, \gamma_{c}\right)$ be the tangent stiffness matrix at the critical point and let $\boldsymbol{\phi}_{c}$ be the

16 eigenvector corresponding to the zero eigenvalue, i.e. $\boldsymbol{K}_{T}\left(\boldsymbol{u}_{c}, \gamma_{c}\right) \boldsymbol{\phi}_{c}=\mathbf{0}$. In the simplest case of

17 the so-called the linearized critical point analysis, the $\boldsymbol{K}_{T}\left(\boldsymbol{u}_{c}, \gamma_{c}\right)$ is approximated as

$\boldsymbol{K}_{T}\left(\boldsymbol{u}_{c}, \gamma_{c}\right) \approx \boldsymbol{K}_{0}+\lambda \boldsymbol{K}_{\sigma}$

18 where $\boldsymbol{K}_{0}$ is the initial stiffness matrix, $\boldsymbol{K}_{\sigma}$ is the geometric stiffness matrix and $\lambda$ is the load

19 factor such that

$$
\begin{aligned}
& \left(\boldsymbol{K}_{0}+\lambda \boldsymbol{K}_{\sigma}\right) \boldsymbol{\phi}=\mathbf{0} \text { or } \\
& \boldsymbol{K}_{0} \boldsymbol{\phi}=-\lambda \boldsymbol{K}_{\sigma} \boldsymbol{\phi}
\end{aligned}
$$

20 The smallest eigenvalue, $\lambda_{1}$, of the generalized eigenvalue problem Eq. (24) is then used to 21 approximate the critical load factor, i.e. $\lambda_{c} \approx \lambda_{1}$ and $\boldsymbol{P}_{c} \approx \lambda_{1} \boldsymbol{P}$. The linear critical point analyses 
1 can provide accurate results in the case where deformations are small and in such cases, linear

2 structural models are adequate. However, the linear critical point analysis usually overestimates

3 the critical load factors for structures undergoing finite deformations/displacements [41], as

4 shown later by an example. In the nonlinear case, to get a better approximation of the critical

$5 \quad$ load factor, $\boldsymbol{K}_{T}\left(\boldsymbol{u}_{c}, \gamma_{c}\right)$ can be approximated as

$\boldsymbol{K}_{T}\left(\boldsymbol{u}_{n}, \gamma_{n}\right) \approx \boldsymbol{K}_{0}+\lambda\left(\boldsymbol{K}_{u}\left(\boldsymbol{u}_{n}, \gamma_{n}\right)+\boldsymbol{K}_{\sigma}\left(\boldsymbol{u}_{n}, \gamma_{n}\right)\right)$

6 where the approximation is carried out at the $n^{\text {th }}$ converged load step. The generalized

7 eigenvalue problem is now given by

$\boldsymbol{K}_{0} \boldsymbol{\phi}=-\lambda\left(\boldsymbol{K}_{u}\left(\boldsymbol{u}_{n}, \gamma_{n}\right)+\boldsymbol{K}_{\sigma}\left(\boldsymbol{u}_{n}, \gamma_{n}\right)\right) \boldsymbol{\phi}$

8 Similar to the linear critical point analysis, the smallest eigenvalue, $\lambda_{1}$, of the generalized

9 eigenvalue problem Eq. (26) is the critical load factor over the base state $\gamma_{n}$. The critical load

10 factor is then approximated as

$\gamma_{c} \approx \lambda_{1} \gamma_{n}$

11 Since only the smallest eigenvalue in Eq. (26) is of interest, instead of directly solving Eq. (26), a

12 better posed generalized eigenvalue problem is solved to obtain the smallest positive eigenvalue

$13 \bar{\lambda}_{1}$ of Eq. (28) as follows [42]

$\boldsymbol{K}_{T}\left(\boldsymbol{u}_{n}, \gamma_{n}\right) \boldsymbol{\phi}_{1}=\bar{\lambda}_{1} \boldsymbol{K}_{0} \boldsymbol{\phi}_{1}$

14 and the smallest eigenvalue $\lambda_{1}$ in Eq. (26) is then given by

$\lambda_{1}=\frac{1}{1-\bar{\lambda}_{1}}$

15 Therefore, based on the critical load factor at $\gamma_{n}$, if $\lambda_{1}<1$ the critical point has been passed and

$16 \gamma_{c}<\gamma_{n}$, while if $\lambda_{1}>1$ the critical point has not been crossed and $\gamma_{c}>\gamma_{n}$. As the stability of an

17 equilibrium solution usually changes at a critical point, $\lambda_{1}$ is a good indicator of stability and can

18 be used to approximate the critical load factor $\gamma_{c}$. 


\subsection{Two bar truss example}

2 To demonstrate the ideas mentioned above in both nonlinear structural analysis and critical load

3 factor analysis, a two-bar truss structure under vertical loading as shown in Figure 2(a) is studied.

4 Each truss member has the length $L=200 \mathrm{~cm}$, cross sectional area $A_{0}=2.5 \mathrm{~cm}^{2}$ and Young's

5 modulus $E=210 \mathrm{GPa}$. This problem is solved using arc-length and secondary path switching

6 method to track the equilibrium solution branches as shown in Figure 2(b).

7 It is observed from Figure 2(b) that this truss structure has the first critical point $A$ at a load of

$812578 \mathrm{kN}$, which is a bifurcation point. After passing the bifurcation point $A$, the load on the

9 primary solution path increases until the limit point $B$ is reached at $15043 \mathrm{kN}$, while the load on the secondary solution branch drops down. The solution path before the critical point $A$ is stable,

11 but both the primary and secondary branches become unstable after passing the critical point $A$,

12 with negative eigenvalues occurring in the tangent stiffness matrix $\boldsymbol{K}_{T}$. This means if a load

13 between $12578 \mathrm{kN}$ and $15043 \mathrm{kN}$ is applied, the load control NR method may still converge but

14 to an unstable primary equilibrium solution. However, only the stable equilibrium path before

15 the critical point $A$ is desirable for the topology optimization process.

The first critical load at point $A$ is predicted by both the linear and nonlinear critical load

17 analyses. With the linear critical load analysis, the predicted critical load is $16996 \mathrm{kN}$, which is 18 much higher than the actual critical load of $12578 \mathrm{kN}$. Therefore, the critical load is 19 overestimated if the linear critical load analysis is used, as discussed earlier. On the other hand, if 20 the nonlinear critical load analysis is employed, the predicted critical loads depend on the state at 21 which the critical load analysis is carried out. As shown in Figure 2, different critical loads $\left(\gamma_{c}\right)$ 22 of $15782 \mathrm{kN}, 14144 \mathrm{kN}$ and $12945 \mathrm{kN}$ are obtained at different load levels $\left(\gamma_{n}\right)$ of $5000 \mathrm{kN}$, $2310000 \mathrm{kN}$ and $12500 \mathrm{kN}$, respectively. Therefore, if the analysis is carried out at $\gamma_{n}=12500 \mathrm{kN}$, 
1 the critical load level is close to the exact value of $12578 \mathrm{kN}$. This exercise demonstrates that

2 with the nonlinear critical load analysis, the closer the load $\left(\gamma_{n}\right)$ gets to the critical load $\left(\gamma_{c}\right)$, the

3 better the critical load prediction becomes. This example also shows the effectiveness of arc-

4 length method, secondary path switching method and nonlinear critical load analysis described in 5 previous sections.

6

7 8 (a)

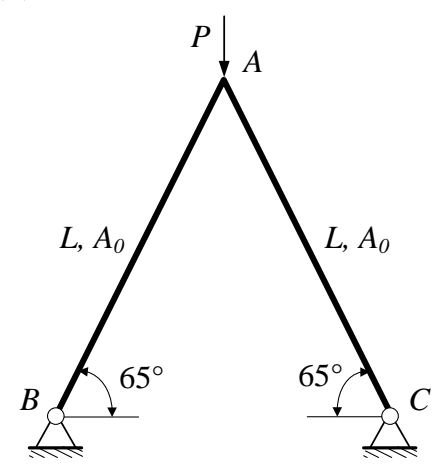

(b)

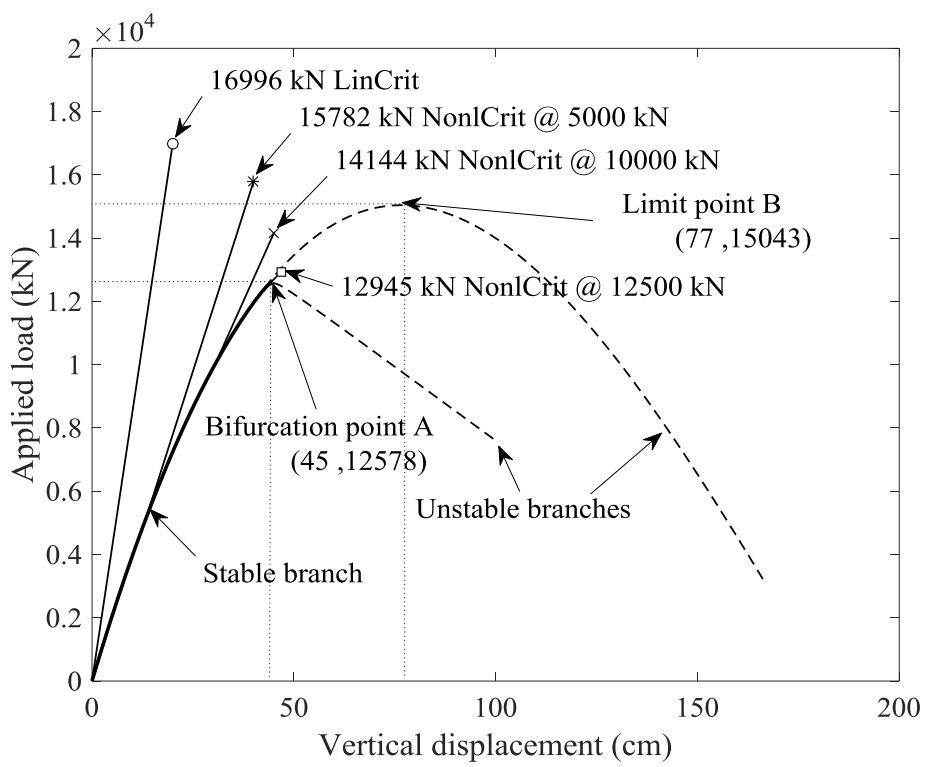

Figure 2. Two-bar truss example: (a) Problem configuration; (b) Structural response and critical point analysis results.

\section{Topology Optimization Formulations and Sensitivity Analyses}

\subsection{Ground structure}

In the ground structure approach, the truss topology optimization aims to find the best cross sectional area and location of each member within a fixed design domain with prescribed member connectivity. Thus, a ground structure consisting of an initial mesh with all potential members is first generated, and the inefficient members are eliminated from the design domain during the optimization process. For the same design domain, the density of ground structure mesh can be different. For instance, in the 2D case, the first difference is the basic node mesh of the domain in $x$ and $y$ direction, such as $5 \times 5$ and $9 \times 9$ node mesh shown in Figure 3(a) and 
1 Figure 3(b), respectively. The second difference is the member connection level, which means

2 how far one node can reach to another node. For the same node mesh, the connection level will

3 determine the complexity of the ground structure as shown in Figure 3(a), Figure 3(c) and Figure

4 3(d). In general, different ground structures will generate different results, as the optimization

5 space expands with increase in the complexity of the ground structure. However, increasing the

6 complexity of the ground structure will also increase the computational effort, which maybe

7 important for large-scale nonlinear truss topology optimization.

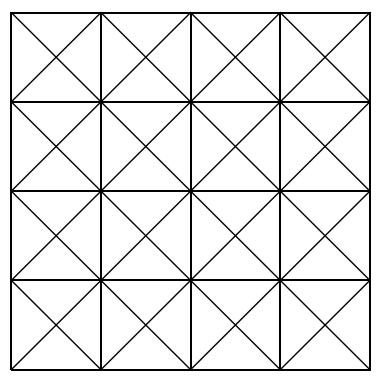

(a)

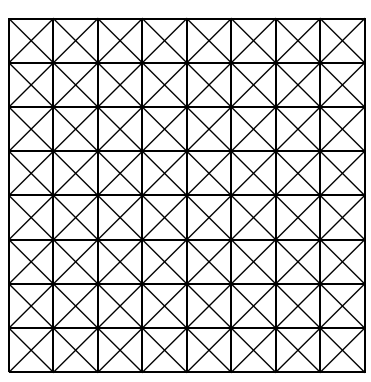

(b)

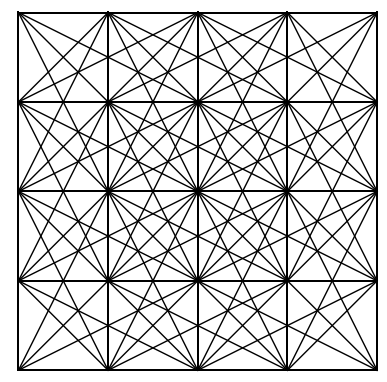

(c)

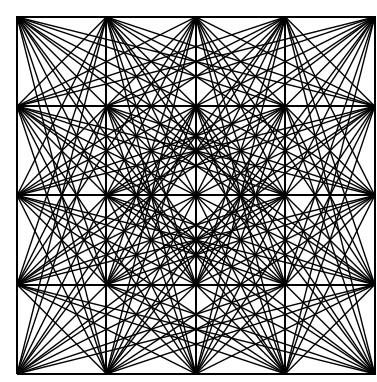

(d)
9 10

11

12

13

14

$$
\begin{array}{ll}
\min _{\boldsymbol{x}} & f_{0}(\boldsymbol{x})=\boldsymbol{P}^{T} \boldsymbol{u}(\boldsymbol{x}) \\
\text { s.t. } & f_{1}(\boldsymbol{x})=\frac{1}{V_{f}} \sum_{e=1}^{n_{e l}} L_{0 e} A_{0 e}-1 \leq 0 \\
& \mathbf{0}<\boldsymbol{x}_{\min } \leq \boldsymbol{x} \leq \boldsymbol{x}_{\max }
\end{array}
$$

\subsection{Compliance formulation}

In the compliance design formulation, a truss system compliance is minimized subjected to the specified loads, volume constraint and box constraints on the design variables. This formulation is given as

where the member cross-sectional areas are taken as the design variables i.e. $x_{e}=A_{0 e}$ and $16 x \in \mathbb{R}^{n_{e l}} ; n_{e l}$ is the total number of truss members; $f_{0}(\boldsymbol{x})$ represents the end compliance of the 17 system; $\boldsymbol{P}$ is the applied load and $\boldsymbol{u}$ is nodal displacement vector; $L_{0 e}$ is the initial length of the 
$1 e^{\text {th }}$ truss member; $f_{1}(\boldsymbol{x})$ represents the volume constraint and $V_{f}$ is the total prescribed material

2 volume; $\boldsymbol{x}_{\min }$ and $\boldsymbol{x}_{\max }$ are the lower and upper limits on the design variable $\boldsymbol{x}$. To avoid

3 numerical difficulties, $\boldsymbol{x}_{\min }$ is set as a small positive number. For solution of the constrained

4 optimization problem in Eq. (30) a nested formulation is adopted [6], wherein at each

5 optimization iteration the displacement $\boldsymbol{u}$ is obtained by solving the equilibrium equations in

6 residual form (Eq. (10)). For structural analyses, the loads are applied using the load control

7 strategy discussed in Section 2.3. As the loads and displacements at the last step are used for the

8 calculating the objective function $f_{0}(\boldsymbol{x})$ in Eq. (30), this formulation is also referred as end

9 compliance. A potential drawback of the above formulation is that the solution may converge to

10 unstable equilibrium branches and the final optimal topologies may be unstable. In addition, as

11 the limit load (if present) of the topologies will evolve during the optimization iterations, the NR

12 process will fail to converge if the applied load is higher than the limit load. Thus, the

13 optimization problem may potentially be ill posed for the given loads and problem settings.

\section{$14 \quad 4.3$ Compliance formulation with critical load constraint}

15 To address stability issue in the above compliance formulation and to ensure that the final 16 optimal topologies are stable, an additional constraint is added to the optimization formulation

17 (Eq. (30)) as follows

$$
\begin{array}{ll}
\min _{\boldsymbol{x}} & f_{0}(\boldsymbol{x})=\boldsymbol{P}^{T} \boldsymbol{u}(\boldsymbol{x}) \\
\text { s.t. } & f_{1}(\boldsymbol{x})=\frac{1}{V_{f}} \sum_{e=1}^{n_{e l}} L_{0 e} A_{0 e}-1 \leq 0 \\
& f_{2}(\boldsymbol{x})=\lambda_{c r}-\lambda_{1}(\boldsymbol{x}) \leq 0 \\
& \mathbf{0}<\boldsymbol{x}_{\min } \leq \boldsymbol{x} \leq \boldsymbol{x}_{\max }
\end{array}
$$

In Eq. (31), $f_{2}(\boldsymbol{x})$ is the additional critical load factor constraint, in which the minimum critical

19 load factor, $\lambda_{1}(\boldsymbol{x})$, is constrained to be greater than a predefined number $\lambda_{c r}$. Moreover, $\lambda_{1}(\boldsymbol{x})$ is 
1 evaluated at the final load step, i.e. for $\gamma_{n}=1.0$, and this constraint ensures that the critical load

2 is always higher than the applied load, and thus it enforces stability of the final topology. A

3 similar critical load factor constraint has been used in the context of linear truss topology

4 optimization in Refs. [17, 21].

\section{$5 \quad 4.4$ Sensitivity analysis}

6 The adjoint method is used to calculate the sensitivity of the objective function [43, 44], and to

7 this end, an auxiliary function $\widetilde{f}_{0}(\boldsymbol{x})$ is defined by premultiplying the equilibrium Eq. (15) with

8 the Lagrangian multipliers $\boldsymbol{\mu}$ and appending it to $f_{0}(\boldsymbol{x})$ as

$\widetilde{f}_{0}(\boldsymbol{x})=f_{0}(\boldsymbol{x})-\boldsymbol{\mu}^{T}\left(\boldsymbol{F}_{\text {int }}(\boldsymbol{u}, \boldsymbol{x})-\boldsymbol{P}\right)$

9 Now taking the derivatives of the above equation with respect to design variable $x_{e}$ yields

$$
\frac{d \widetilde{f}_{0}(\boldsymbol{x})}{d x_{e}}=\frac{d f_{0}(\boldsymbol{x})}{d x_{e}}=\left(\boldsymbol{P}^{T}-\boldsymbol{\mu}^{T} \boldsymbol{K}_{T}\right) \frac{d \boldsymbol{u}}{d x_{e}}-\boldsymbol{\mu}^{T} \frac{d \boldsymbol{F}_{i n t}}{d x_{e}}=\mathbf{0}
$$

10 The derivatives $d \boldsymbol{F}_{\text {int }} / d x_{e}$ can be explicitly expressed and obtained during the finite element

11 assembly process. To avoid calculation of the implicit derivatives $d \boldsymbol{u} / d x_{e}$, the Lagrangian

12 multipliers are determined by the solution of the adjoint system

$\boldsymbol{\mu}^{T} \boldsymbol{K}_{T}^{T}=\boldsymbol{P}^{T}$

13 where $\boldsymbol{K}_{T}^{T}=\boldsymbol{K}_{T}$. Therefore, the sensitivity of the end compliance $f_{0}(\boldsymbol{x})$ is

$\frac{d f_{0}(\boldsymbol{x})}{d x_{e}}=-\boldsymbol{\mu}^{T} \frac{d \boldsymbol{F}_{i n t}}{d x_{e}}$

14 Next, the sensitivity of the volume constraint $f_{1}(\boldsymbol{x})$ is given by

$\frac{d f_{1}(\boldsymbol{x})}{d x_{e}}=\frac{L_{0 e}}{V_{f}}$

15 For determining the sensitivity of the critical load factor constraint $f_{2}(\boldsymbol{x})$, the following 16 eigenvalue analysis is considered 


$$
\left[\boldsymbol{K}_{0}(\boldsymbol{x})+\lambda_{1} \boldsymbol{K}_{u}(\boldsymbol{u}, \boldsymbol{x})+\lambda_{1} \boldsymbol{K}_{\sigma}(\boldsymbol{u}, \boldsymbol{x})\right] \phi_{1}=\mathbf{0}
$$

1 To avoid the calculation of implicit derivatives $d \boldsymbol{\phi}_{1} / d x_{e}$ and $d \boldsymbol{u} / d x_{e}$, the adjoint formulation is

2 constructed by premultiplying Eq. (37) by $\boldsymbol{\phi}_{1}^{T}$ and appending equilibrium equations (Eq. (15))

$3 \quad$ using Lagrangian multipliers $\boldsymbol{\mu}$ as

$\boldsymbol{\phi}_{1}^{T}\left[\boldsymbol{K}_{0}(\boldsymbol{x})+\lambda_{1} \boldsymbol{K}_{u}(\boldsymbol{u}, \boldsymbol{x})+\lambda_{1} \boldsymbol{K}_{\sigma}(\boldsymbol{u}, \boldsymbol{x})\right] \boldsymbol{\phi}_{1}-\boldsymbol{\mu}^{T}\left[\boldsymbol{F}_{\text {int }}(\boldsymbol{u}, \boldsymbol{x})-\boldsymbol{P}\right]=\mathbf{0}$

4 Taking the derivatives of above equation with respect to design variable $x_{e}$ and gathering the

5 terms containing $d \boldsymbol{u} / d x_{e}$ yields

$$
\begin{aligned}
\boldsymbol{\phi}_{1}^{T}\left[\frac{d \boldsymbol{K}_{0}}{d x_{e}}+\lambda_{1}(\right. & \left.\left.\frac{d \boldsymbol{K}_{u}}{d x_{e}}+\frac{d \boldsymbol{K}_{\sigma}}{d x_{e}}\right)+\frac{d \lambda_{1}}{d x_{e}}\left(\boldsymbol{K}_{u}+\boldsymbol{K}_{\sigma}\right)\right] \boldsymbol{\phi}_{1} \\
+ & {\left[\lambda_{1} \boldsymbol{\phi}_{1}^{T}\left(\frac{\partial \boldsymbol{K}_{u}}{\partial \boldsymbol{u}}+\frac{\partial \boldsymbol{K}_{\sigma}}{\partial \boldsymbol{u}}\right) \boldsymbol{\phi}_{1}-\boldsymbol{\mu}^{T} \boldsymbol{K}_{T}\right] \frac{d \boldsymbol{u}}{d x_{e}}-\boldsymbol{\mu}^{T} \frac{d \boldsymbol{F}_{\text {int }}}{d x_{e}}=\mathbf{0} }
\end{aligned}
$$

6 To avoid calculation of $d \boldsymbol{u} / d x_{e}$, the Lagrangian multipliers $\boldsymbol{\mu}$ are determined using the

7 following adjoint system

$\boldsymbol{\mu}^{T} \boldsymbol{K}_{T}^{T}=\lambda_{1} \boldsymbol{\phi}_{1}^{T}\left(\frac{\partial \boldsymbol{K}_{u}}{\partial \boldsymbol{u}}+\frac{\partial \boldsymbol{K}_{\sigma}}{\partial \boldsymbol{u}}\right) \boldsymbol{\phi}_{1}$

8 where $\partial \boldsymbol{K}_{u} / \partial \boldsymbol{u}$ and $\partial \boldsymbol{K}_{\sigma} / \partial \boldsymbol{u}$ are the $3^{\text {rd }}$ order tensors that are obtained analytically from Eq. (12)

9 to Eq. (14). Finally, the sensitivities of the critical load factor are obtained as

$$
\frac{d \lambda_{1}}{d x_{e}}=\frac{\boldsymbol{\mu}^{T} \frac{d \boldsymbol{F}_{i n t}}{d x_{e}}-\boldsymbol{\phi}_{1}^{T}\left[\frac{d \boldsymbol{K}_{0}}{d x_{e}}+\lambda_{1}\left(\frac{d \boldsymbol{K}_{u}}{d x_{e}}+\frac{d \boldsymbol{K}_{\sigma}}{d x_{e}}\right)\right] \boldsymbol{\phi}_{1}}{\boldsymbol{\phi}_{1}^{T}\left(\boldsymbol{K}_{u}+\boldsymbol{K}_{\sigma}\right) \boldsymbol{\phi}_{1}}
$$

\section{Localized Modes Issue}

11 Spurious localized modes is an another issue that has to be addressed in topology optimization 12 considering the eigenvalue analysis [31, 32]. Unlike size or shape optimization, the topology 13 optimization process generates low-density and solid elements. For instance, in density based 14 continuum topology optimization low-density elements are termed as void elements [45], while 
1 in truss topology optimization the members with the cross sectional area below certain threshold

2 are considered as low-density or slim bar elements, and are removed from the final topology

3 results. The solid elements are the only ones that are present in the final optimal topologies. The

4 low-density elements have little effect on the objective function $f_{0}(\boldsymbol{x})$, however, these elements

5 influence the results of eigenvalue analysis and should be properly addressed during the 6 optimization process.

7 To illustrate the localized modes issue, a 28-bar truss example as shown in Figure 4 is studied.

8 The square domain in Figure 4 has $3 \times 3$ nodes ground structure with all the potential connections.

9 The applied load is $P=50 \mathrm{kN}$, Young's modulus is $E=210 \mathrm{GPa}$ and the domain length is $L=$ $10100 \mathrm{~cm}$. The solid bars $A_{16}$ and $A_{67}$ have cross sectional area of $1 \mathrm{~cm}^{2}$, while the slim bars $A_{45}$ 11 and $A_{56}$ have cross sectional area of $10^{-6} \mathrm{~cm}^{2}$. All of the other slim bars have cross sectional area 12 of $10^{-9} \mathrm{~cm}^{2}$. The threshold area for removing the bars is set to $10^{-3} \mathrm{~cm}^{2}$ so that only nodes 1,6 13 and 7 are "true" nodes, i.e. the nodes that connect the solid elements, while the nodes $2,3,8$, and 149 are the "spurious" nodes as they connect only the low-density elements. The structure is solved 15 using 5 load control steps and the critical load analysis is carried out based on the last step of the nonlinear analysis.

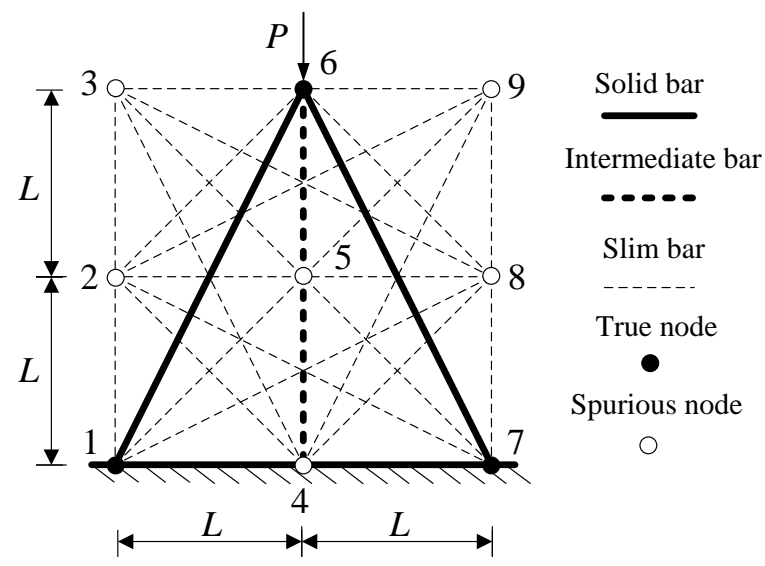


1 The first three eigenvalues and eigenvectors of the generalized eigenvalue problem (Eq. (28)) are

2 computed and presented in Figure 5. The first eigenmode shown in Figure 5(a) corresponds to

3 the localized deformation in the slim or low-density elements, and is termed as pseudo or

4 spurious mode. The eigenvalue of $\lambda_{1}=0.67$ corresponding to this mode should not be used to

5 formulate the constraint in Eq. (31) as there is little displacement of the true node (node 6) and

6 most of displacement occurs at spurious nodes (2, 3, 8 and 9). The second and third smallest

7 eigenvalues $\lambda_{2}=150.01$ and $\lambda_{3}=200.02$ are much higher than $\lambda_{1}$, and the corresponding

8 modes are shown in Figure 5(b) and Figure 5(c), respectively. For these two modes, there is

9 considerable displacement of the true nodes and these two modes are the true eigenmodes, i.e.

10 the modes corresponding to the deformation in the solid members. To determine the correct

11 critical load, the critical load analysis on the final topology without the slim bars is carried out,

12 and the two critical modes are depicted in Figure 6(a) and Figure 6(b), respectively. It can be

13 observed that the true critical modes are the second and third modes when slim bars are

14 considered with the true critical load factors of $\lambda_{1}^{*}=150.01$ and $\lambda_{2}^{*}=200.02$. Therefore, if the

15 smallest critical load factor $\lambda_{1}=0.67$ is used in topology optimization, it will not represent the

16 true critical mode for the final topology. This exercise shows that the eigenmodes corresponding

17 to the low-density elements are not important and only the eigenmodes associated with solid

18 elements are of practical interest.

19 To address the localized modes for the nonlinear truss topology optimization, a new scheme 20 based on the spurious modal strain energy ratio is proposed. In this approach, the spurious nodes

21 in the topology are first determined based on the specified area threshold for the slim bars. The

22 degrees of freedom related to these spurious nodes are then identified and the $r^{\text {th }}$ eigenvector 
1 associated with the eigenvalue $\lambda_{r}$ of the generalized eigenvalue problem (Eq. (28)) is

2 decomposed as

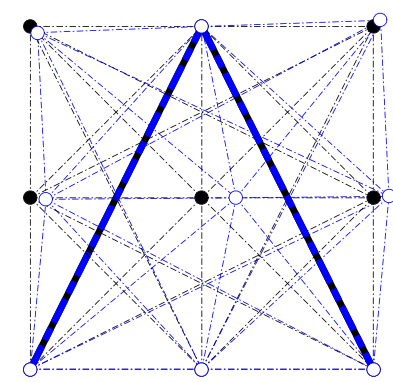

(a) $\lambda_{1}=0.67$

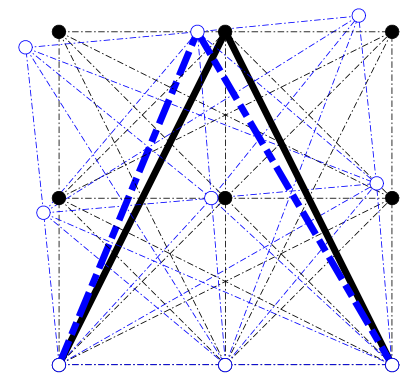

(b) $\lambda_{2}=150.01$

$E S_{2}=7.29 \times 10^{-9}$

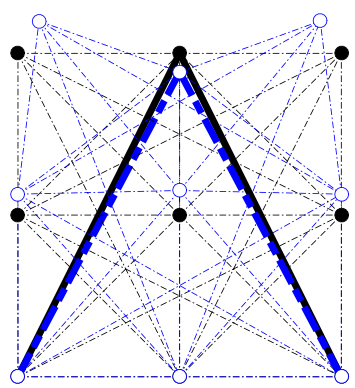

(c) $\lambda_{3}=200.02$ $E S_{3}=6.72 \times 10^{-6}$
6

7

$\phi_{r}=\phi_{r_{t}}+\phi_{r_{s}}$

8

11 The spurious modal energy ratio (Eq. (43) $)_{1}$ gives the ratio of the energy stored in the slim bars

12 to the total energy in the $r^{\text {th }}$ eigenmode. If the spurious modal energy ratio for a particular mode 13 is close to 1.0 then that mode can be considered as a spurious mode and if the spurious modal

where the vector $\boldsymbol{\phi}_{r_{t}}$ corresponds to the degrees of freedom associated with the true nodes and $\boldsymbol{\phi}_{r_{s}}$ corresponds to the degrees of freedom associated with the spurious nodes. The spurious modal strain energy ratio associated with the $r^{\text {th }}$ eigenmode is then defined as

$$
\begin{aligned}
& E S_{r}=\frac{\boldsymbol{\phi}_{r_{s}}^{T} \boldsymbol{K}_{T} \boldsymbol{\phi}_{r}}{\boldsymbol{\phi}_{r}^{T} \boldsymbol{K}_{T} \boldsymbol{\phi}_{r}} \\
& 0<E S_{r}<1
\end{aligned}
$$$$
\begin{array}{ll}
\text { (a) } \lambda_{1}^{*}=150.01 & \text { (b) } \lambda_{2}^{*}=200.02
\end{array}
$$

Third mode.

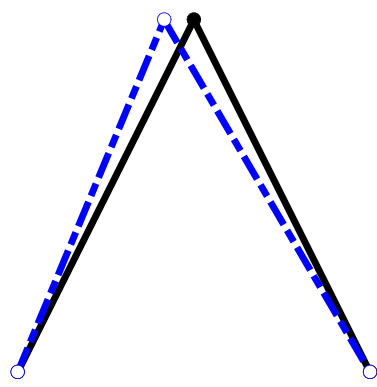

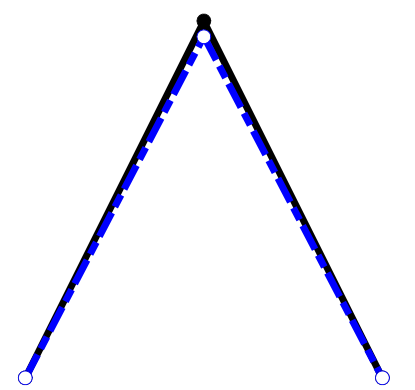


energy ratio is small then it is a true mode. To demonstrate this idea, the $E S_{r}$ values of different modes for the 28-bar truss problem are also shown in Figure 5. It is observed that for the first mode the ratio is $E S_{1}=0.99$, which means this mode is a spurious mode, while for the second and third modes $E S_{2}=7.29 \times 10^{-9}$ and $E S_{3}=6.72 \times 10^{-6}$ which indicate that these modes are true modes, i.e. the modes in which there is significant deformation in the solid elements. This conclusion is also validated by the checking the eigenmodes without slim bars as shown in Figure 6. Usually, if the design variables are close to 0 and $A_{\max }$, the $E S_{r}$ values for each mode will approach 0 and 1 . However, as the intermediate areas are also present, the $E S_{r}$ values will range from 0 to 1 for different eigenmodes. Therefore, to identity spurious modes a threshold value $E S_{T}$ of spurious modal energy is defined, and if $E S_{r}$ is greater than $E S_{T}$ then the associated eigenmode is considered to be a spurious mode. In this study, the threshold value of $E S_{T}=0.8$ is used to identify the true eigenmodes and the corresponding eigenvalues. The minimum eigenvalue that is associated with a true eigenmode is then used to formulate the critical load factor constraint in Eq. (31).

\section{Numerical Examples}

Three examples are presented in this section to investigate the effectiveness of the proposed formulations for solving nonlinear truss topology optimization. For all the problems, Young's modulus is set to $E=210 \mathrm{GPa}$ and $E S_{T}=0.8$, with the slim bar area threshold of $10^{-3} \mathrm{~cm}^{2}$. The initial design $\left(\boldsymbol{x}^{0}\right)$ is set as the ratio of the total available material volume to the sum of all the potential bar lengths in the ground structure. The upper bound for member area is $x_{e}^{\max }=10$ $\mathrm{cm}^{2}$ and the lower bound is $x_{e}^{\min }=10^{-9} \mathrm{~cm}^{2}$. An optimization algorithm based on interior point method [46] is employed as the optimizer and the termination criteria is either the change in the design variable, i.e. $\left\|x^{k+1}-x^{k}\right\|_{2} \leq 10^{-12}$ or change in the objective function, i.e. $\mid f_{0}^{k+1}-$ 
$1 f_{0}^{k} \mid \leq 10^{-12}$, where $k$ is the optimization iteration number. It should be noted that only a local

2 minima is ensured due to the non-convex nature of the problem formulation. Moreover, during

3 the optimization iterations, structural analyses are carried out using the load control strategy

4 described in Section 2.3 with $N=5$, i.e. the load is applied in five equal steps. In the final

5 topology results, the blue color members are in tension and the red color members are in

6 compression, and the line thickness is used to indicate the relative areas. All the implementations

7 and computations in this study are carried out in a Matlab based finite element library CPSSL-

8 FEA developed at the University of Notre Dame.

\subsection{Clamped domain problem}

10 In the first example, a laterally supported rectangular domain subjected to vertical load at top

11 center is studied. The problem configuration and the initial ground structure with $9 \times 3$ nodes and

12 full bar connectivity are shown in Figure 7(a) and Figure 7(b), respectively. The available

13 material volume for the design is limited to $V_{f}=200 \mathrm{~cm}^{3}$ and the domain dimension is $L=100$

$14 \mathrm{~cm}$. Symmetry is enforced along the vertical direction according to the geometry of the problem 15 configuration.

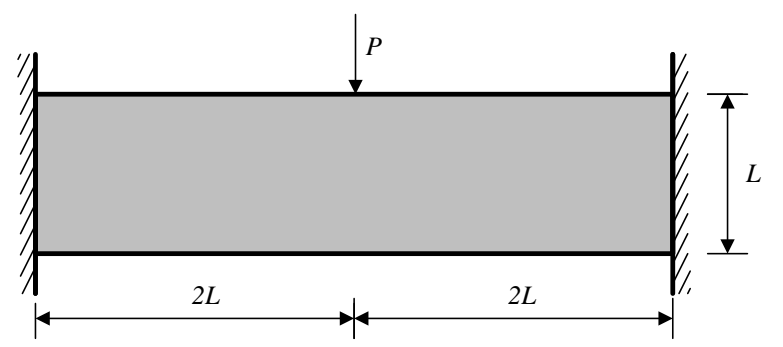

(a)

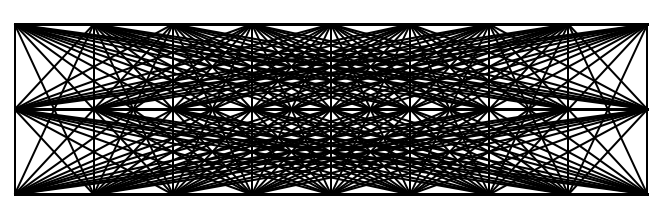

(b)
16

6.1.1 Compliance formulation 
1 This example is first optimized using the compliance formulation (Eq. (30)), and the result of

2 linear elastic case with load of $P=100 \mathrm{kN}$ and of nonlinear cases under different load

3 magnitudes of $P=10 \mathrm{kN}, P=100 \mathrm{kN}$ and $P=200 \mathrm{kN}$ are presented in Figure 8(a), Figure 8(b),

4 Figure 8(c) and Figure 8(d), respectively. As shown in Figure 8(a), with linear elastic model, the

5 optimal topology has two collinear bars under compression to support the applied load and this

6 result is independent of the load magnitude. For the nonlinear model, under a small load

7 magnitude of $P=10 \mathrm{kN}$, the geometrical nonlinearities are insignificant and the topology

8 obtained is similar to the linear elastic case, as shown in Figure 8(b). When the load magnitude is

9 increased to $P=100 \mathrm{kN}$, however, the topology becomes different from the linear elastic case

10 and more material is distributed to the bottom side of the domain to resist the applied load in

11 tension, as shown in Figure 8(c). With further increase in the load magnitude to $P=200 \mathrm{kN}$, the

12 final topology is significantly different and more material is distributed to the members in

13 tension, as shown in Figure 8(d). Despite the differences between the final topologies in the

14 linear and nonlinear cases, topologies generated with the compliance formulation (Eq. (30)) have

15 collinear bars under compression with "un-braced" or "hanging nodes", and this configuration is

16 undesirable from stability considerations, as shown later. Although these hanging nodes can be

17 eliminated by a node merging post-processing step, it will lead to sub-optimal designs and the 18 stability of the final designs is still questionable [18]. 


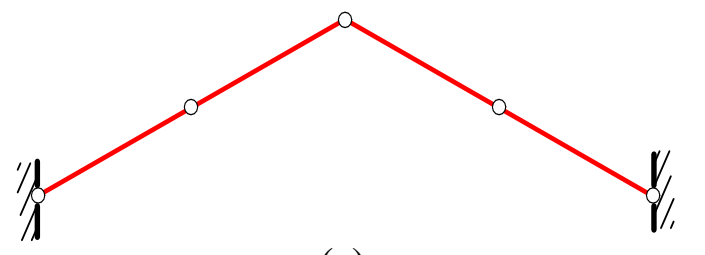

(a)

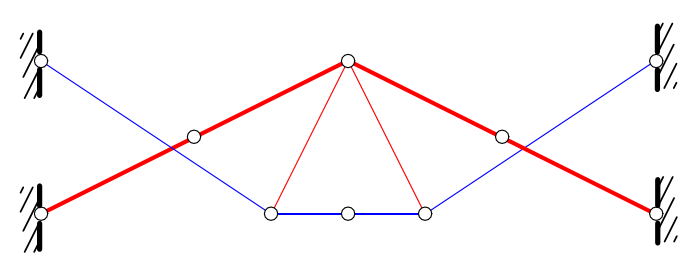

(c)

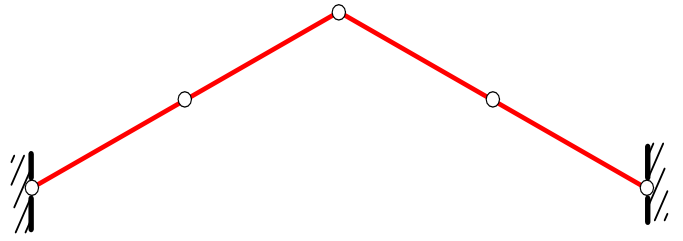

(b)

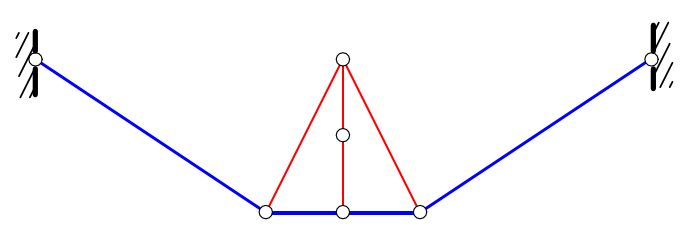

(d)

Figure 8. Clamped domain problem topologies in compliance formulation: (a) Linear elastic; (b) Nonlinear $P=10 \mathrm{kN}$; (c) Nonlinear $P=100 \mathrm{kN}$; (d) Nonlinear $P=200 \mathrm{kN}$.

The first critical load factors for the final topologies shown in Figure 8 are evaluated, and the results together with the first true critical modes are presented in Figure 9. Since the linear and nonlinear case with $P=10 \mathrm{kN}$ have similar topologies, only the nonlinear topology result is shown in Figure 9(a). The solid circles in these figures show the initial node position, while the hollow circles indicate the deformed position. As the first critical load can be approximated as $P_{c} \approx \lambda_{1} P$, from Figure 9 it can be observed that $\lambda_{1}<1.0$ for all the topologies generated using the compliance formulation and the first critical load is much lower than the applied load. Moreover, all the critical modes are true modes as $E S<0.8$ for all the modes. The deformation in these modes is mostly localized in the compression members and is related to the instability in the collinear compression members. Eigenvalue analysis is further carried out to check the stability of the topologies and the results are presented in Table 2. At the initial configuration, the minimum eigenvalue of the tangent stiffness matrices $\left(\boldsymbol{K}_{T}\right)$ are positive indicating that the initial configurations (under zero loads) are stable. Note that the slim bar members with $A \leq 10^{-3} \mathrm{~cm}^{2}$ are not shown in the topologies, and these members contribute to the stability of topologies at zero loads. In the deformed configuration, at the final load application step, the first two 
1 eigenvalues are negative, indicating that this equilibrium state is unstable. The slim bar members

2 are still present but are unable to ensure stability. Thus, the final topologies are unstable if the

3 compliance formulation is used without any stability constraints. In addition, as the stability is

4 associated with the compressive collinear bars, merging the hanging nodes in collinear

5 compression members will influence the stability of the system, while merging the hanging

6 nodes in collinear tension members will have little influence on the stability.

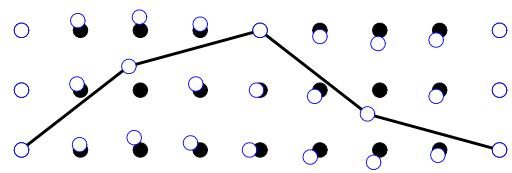

(a) $\lambda_{1}=5.65 \times 10^{-6}$
7

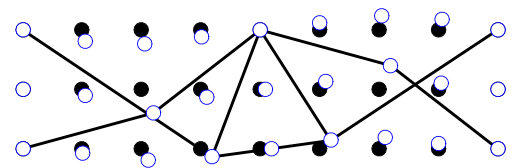

(b) $\lambda_{1}=0.1778$ $E S_{1}=1.27 \times 10^{-4}$

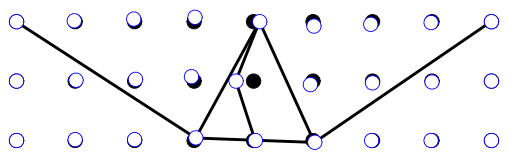

(c) $\lambda_{1}=3.47 \times 10^{-8}$ $E S_{1}=8.47 \times 10^{-6}$

Figure 9. True critical modes for clamped domain problem topologies in compliance formulation: (a) Nonlinear $P=10 \mathrm{kN}$; (b) Nonlinear $P=100 \mathrm{kN}$; (c) Nonlinear $P=200 \mathrm{kN}$.

\begin{tabular}{cccc}
\hline & Initial configuration & \multicolumn{2}{c}{ Final configuration } \\
\hline & First eigenvalue & First eigenvalue & Second eigenvalue \\
\hline Linear elastic & $3.1510 \times 10^{-7}$ & - & - \\
$P=10 \mathrm{kN}$ & $3.1587 \times 10^{-7}$ & $-2.0218 \times 10^{-1}$ & $-2.0127 \times 10^{-1}$ \\
$P=100 \mathrm{kN}$ & $3.8742 \times 10^{-5}$ & $-1.7361 \times 10^{-2}$ & $-1.2549 \times 10^{-2}$ \\
$P=200 \mathrm{kN}$ & $7.1803 \times 10^{-7}$ & $-6.1889 \times 10^{-1}$ & $-1.8018 \times 10^{-2}$ \\
\hline
\end{tabular}

Table 2. Tangent stiffness eigenvalue analysis of clamped domain problem topologies in compliance formulation 


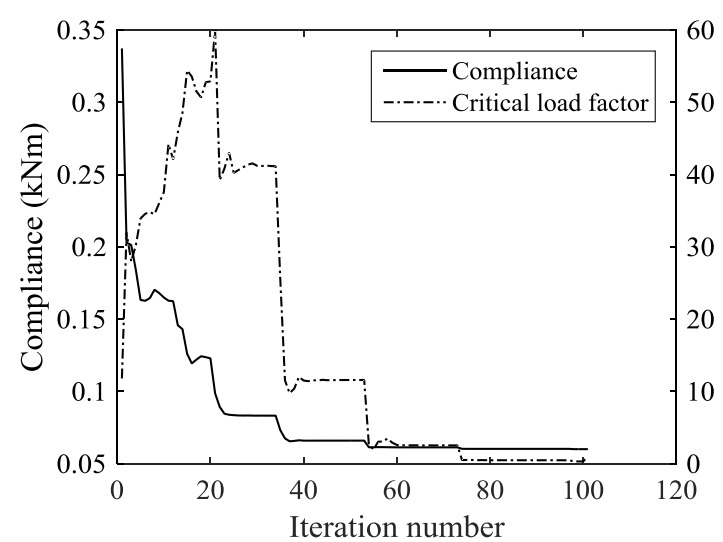

(a)

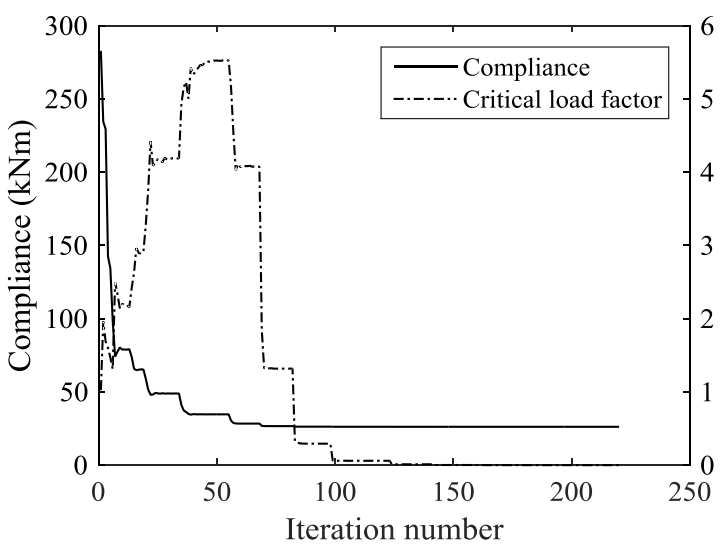

(c)

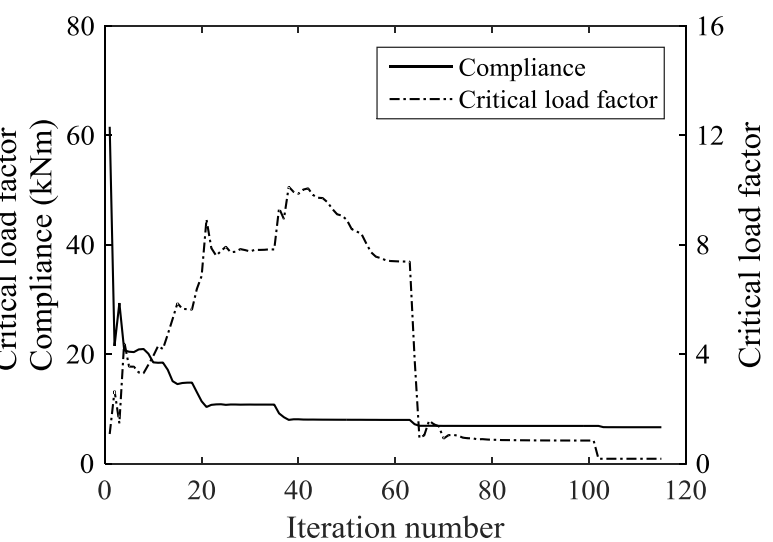

(b)

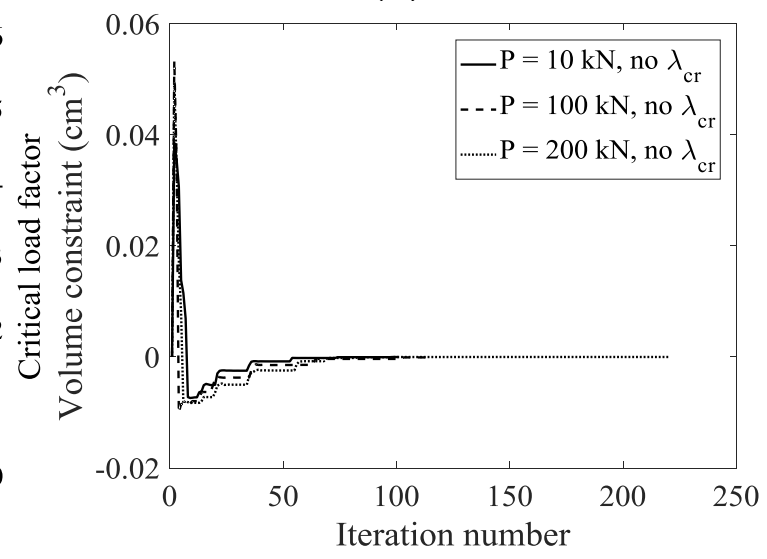

(d)

Figure 10. Iteration history curves of clamped domain problem in compliance formulation: (a) $P$ $=10 \mathrm{kN}$; (b) $P=100 \mathrm{kN}$; (c) $P=200 \mathrm{kN}$; (d) Volume constraints.

4 The convergence histories of the compliance, critical load factor, and volume constraint during

5 optimization process are shown in Figure 10. From Figure 10(a) to Figure 10(c), it can be

6 observed that the compliance decreases during the optimization process, however, the decrease

7 in compliance is non-monotonic due to the non-convexity of the optimization problem. The

8 volume constraint is also satisfied in all cases, as shown in Figure 10(d). Since the stability

9 constraint is not included, the first critical load factor becomes smaller than 1.0, indicating that

10 the stability of topologies is lost during the optimization process. 
1 Topology optimization is again carried out on this problem using the nonlinear model and

2 compliance formulation with critical load constraint (Eq. (31)), where $\lambda_{c r}=1.0$ is prescribed.

3 Topology results for the three different load magnitudes of $P=10 \mathrm{kN}, P=100 \mathrm{kN}$ and $P=200$

$4 \quad \mathrm{kN}$ are shown in Figure 11(a), Figure 11(b) and Figure 11(c), respectively. These results show

5 that with the inclusion of the critical load factor constraint, more truss members are introduced

6 into the final topologies and the hanging nodes in the compressive collinear bars are supported

7 by additional members. Since the critical load associated with instabilities in compressive

8 collinear bars is small, this unstable configuration is stabilized by incorporating the critical load

9 factor constraint. Another important observation is that the collinear bars in tension are still

10 present in the final topologies as shown in Figure 11(a) and Figure 11(b). This is because the

11 collinear tensile bars are stable and these topologies satisfy the critical load constraint, as shown

12 next. The hanging nodes in tensile collinear bars can be merged in the final topology designs

13 without effecting the stability and performance of the structure.

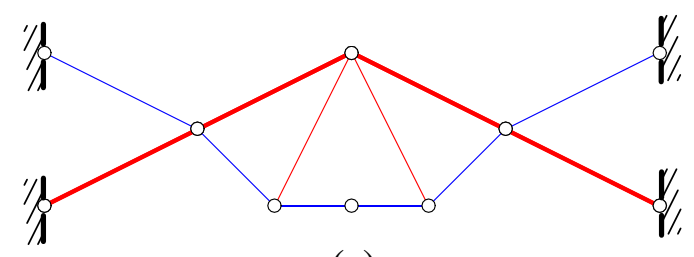

(a)

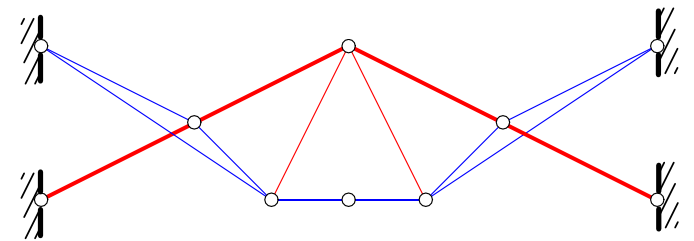

(b)

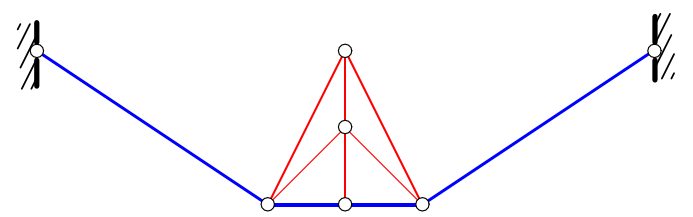

(c)

14

Figure 11. Clamped domain problem topologies in critical load constraint formulation $\left(\lambda_{c r}=1.0\right)$ : (a) Nonlinear $P=10 \mathrm{kN}$; (b) Nonlinear $P=100 \mathrm{kN}$; (c) Nonlinear $P=200 \mathrm{kN}$.

The first three critical modes of topologies in Figure 11 are shown in Figure 12, Figure 13 and Figure 14, respectively, in which the load factors $\lambda_{r}$ and spurious modal energy ratios $E S_{r}$ are also presented. It can be observed that the first three load factors are different for all the cases, 
which implies that these eigenvalues are simple. Furthermore, in the first and third case, the

2 minimum eigenvalue $\lambda_{1}=1.00$ with $E S_{1}<0.8$ for these eigenmodes, indicating that the first

3 mode is the true eigenmode. For the second case, the first eigenvalue is $\lambda_{1}=1.68 \times 10^{-2}$ with

$4 E S_{1}=0.99$, which implies that the first mode is a spurious mode (Figure 13(a)). However, the

5 second eigenvalue is $\lambda_{2}=1.00$ with $E S_{2}=5.46 \times 10^{-7}$ (Figure 13(b)), and thus the second mode

6 is the true mode in this case. Thus, the critical load factor constraint is enforced for the first true

7 mode and these results further validates the spurious/localized mode identification process.

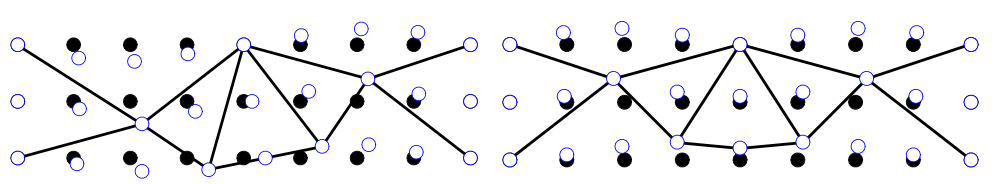

(a) $\lambda_{1}=1.00$ $E S_{1}=3.01 \times 10^{-6}$ (b) $\lambda_{2}=1.62$ $E S_{2}=1.36 \times 10^{-5}$

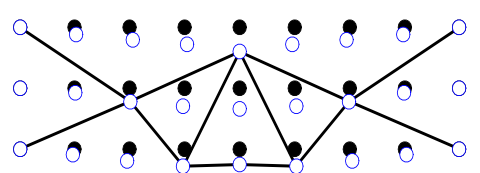

(c) $\lambda_{3}=56.07$ $E S_{3}=1.53 \times 10^{-5}$
Figure 12. Critical modes for clamped domain problem topologies in critical load constraint formulation ( $\left.P=10 \mathrm{kN}, \lambda_{c r}=1\right)$ : (a) First mode; (b) Second mode; (c) Third mode.

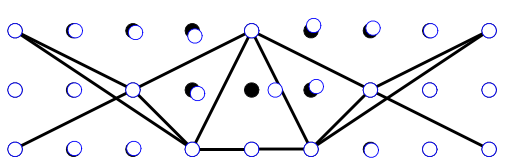

(a) $\lambda_{1}=1.68 \times 10^{-2}$

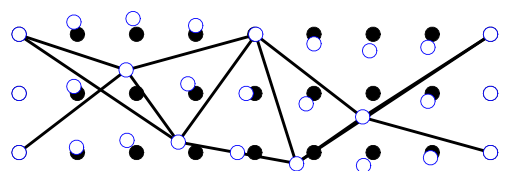

(b) $\lambda_{2}=1.00$

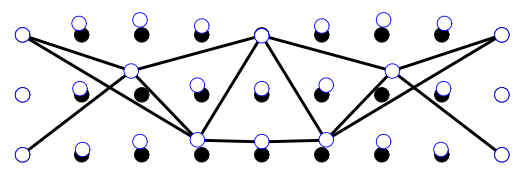

(c) $\lambda_{3}=1.44$

Figure 13. Critical modes for clamped domain problem topologies in critical load constraint formulation ( $\left.P=100 \mathrm{kN}, \lambda_{c r}=1\right)$ : (a) First mode; (b) Second mode; (c) Third mode.

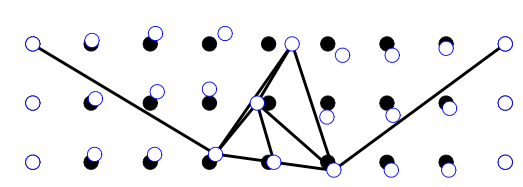

(a) $\lambda_{1}=1.00$

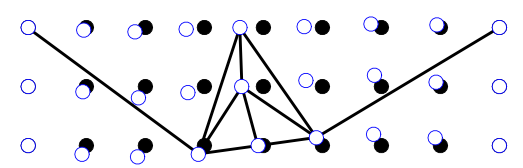

(b) $\lambda_{2}=1.27$

$E S_{2}=2.67 \times 10^{-2}$

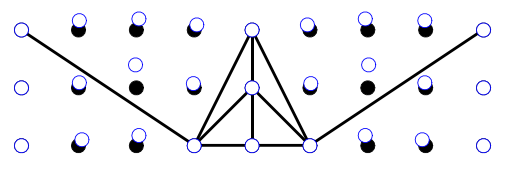

(c) $\lambda_{3}=4.64$

$E S_{3}=0.999$

Figure 14. Critical modes for clamped domain problem topologies in critical load constraint formulation ( $\left.P=200 \mathrm{kN}, \lambda_{c r}=1\right)$ : (a) First mode; (b) Second mode; (c) Third mode. 
1 The convergence histories of the compliance, critical load factor, and volume constraint during

2 the optimization process are shown in Figure 15. It can be observed that for all cases the

3 convergence is achieved within 200 steps and the termination criterion of $\left\|\boldsymbol{x}^{k+1}-\boldsymbol{x}^{k}\right\|_{2} \leq 10^{-12}$

4 is satisfied. The objective function values decrease during the optimization process, however, the

5 decrease is not monotonic due the high non-convexity of the optimization problem. The critical

6 load factors $\lambda_{1}$ first increase and then decrease, and finally converge to the prescribed critical

7 load factor constraint value of $\lambda_{c r}=1.0$. Although the critical load factor constraint is violated at

8 few optimization steps, the final designs satisfy the constraints. These constraint violations are

9 due to the sudden changes in topology during the optimization process as shown by the topology

10 results at iteration steps 90, 91 and 94 for the load case of $P=200 \mathrm{kN}$ (Figure 16). The topology

11 shown in Figure 16(c) is unstable; however, this topology is stabilized as the optimization

12 proceeds. It is also observed that the critical load factor is usually higher and the critical load 13 factor constraint is always satisfied for the smaller load cases $(P=10 \mathrm{kN}$ and $P=100 \mathrm{kN})$ as

14 compared to the larger load case $(P=200 \mathrm{kN})$ where the constraint is violated at few steps. In 15 addition, the volume constraint is enforced during the optimization process as shown in Figure $1615(d)$. 


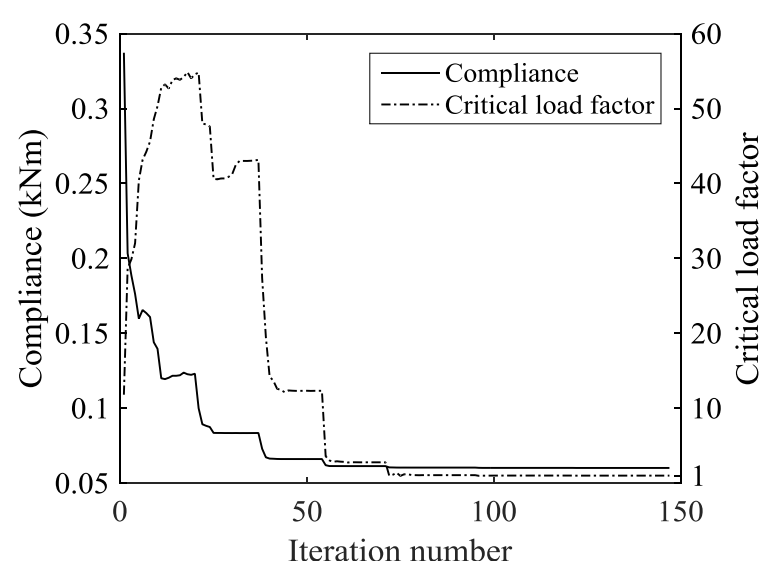

(a)

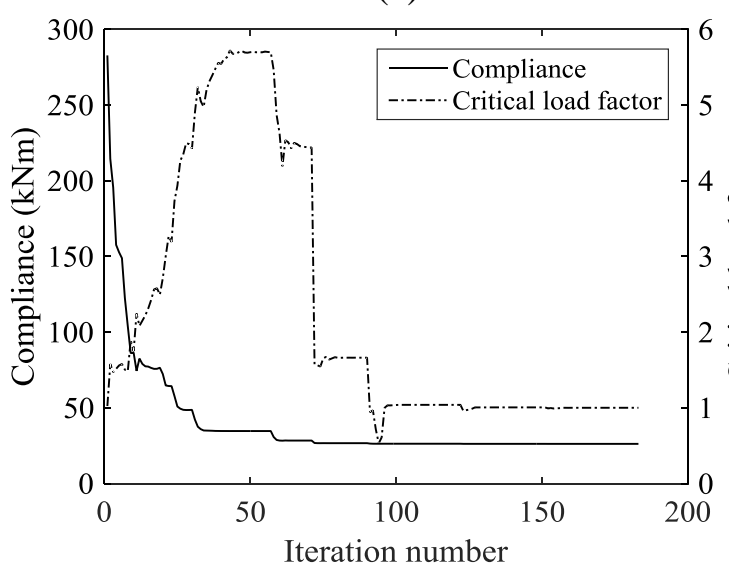

(c)

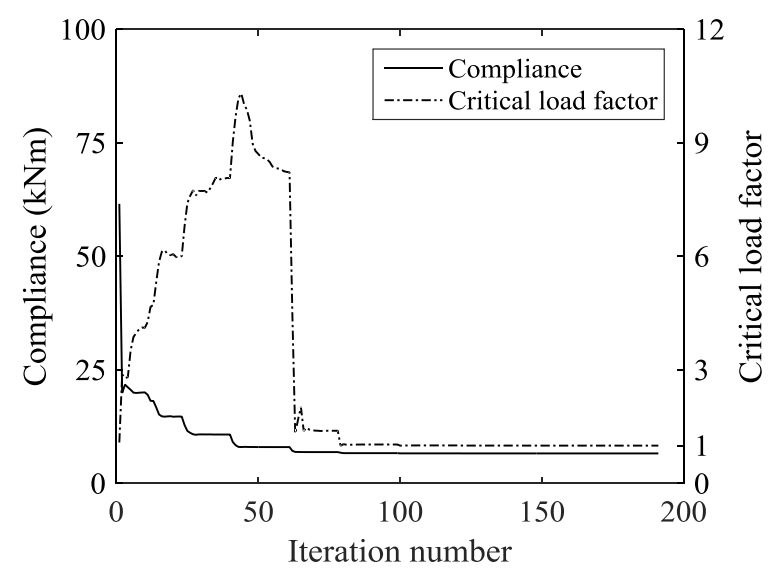

(b)

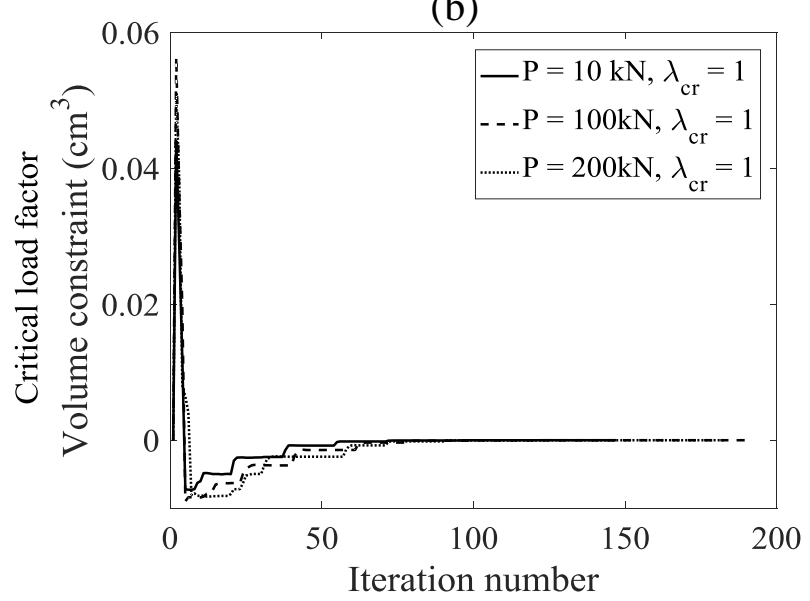

(d)

Figure 15. Iteration history curves of clamped problem in critical load constraint formulation: (a) $P=10 \mathrm{kN}$; (b) $P=100 \mathrm{kN}$; (c) $P=200 \mathrm{kN}$; (d) Volume constraints.

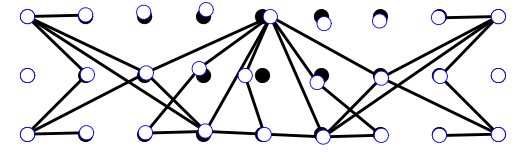

(a) $\lambda_{1}=1.6629$

$E S_{1}=0.3753$

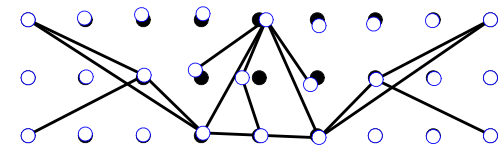

(b) $\lambda_{1}=0.9434$

$E S_{1}=0.1537$

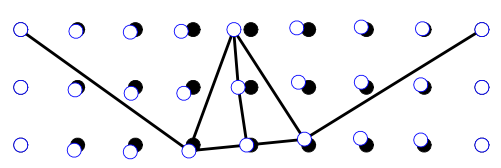

(c) $\lambda_{1}=0.5315$
5

6 7 


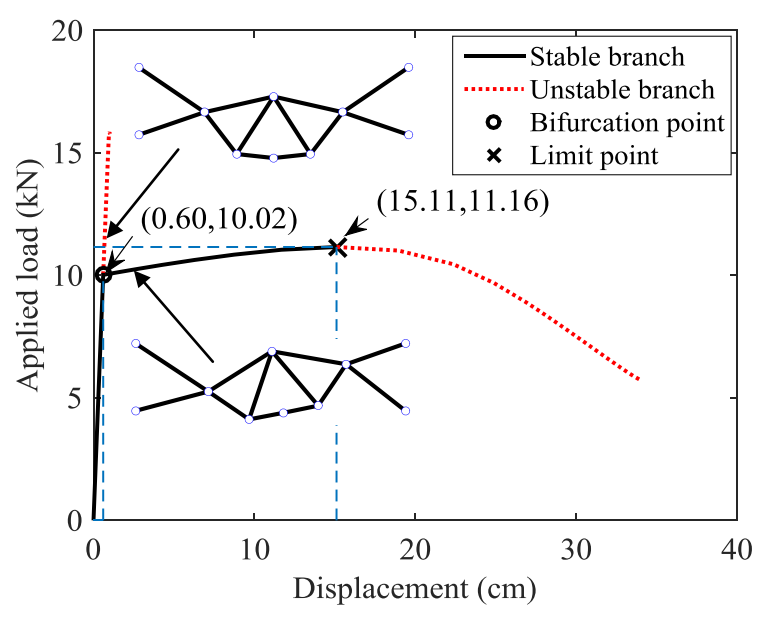

(a)

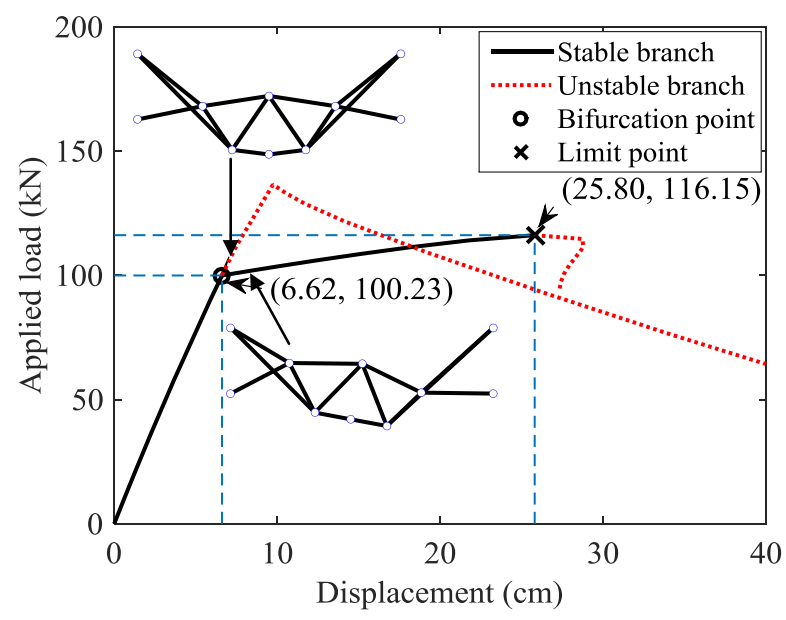

(b)

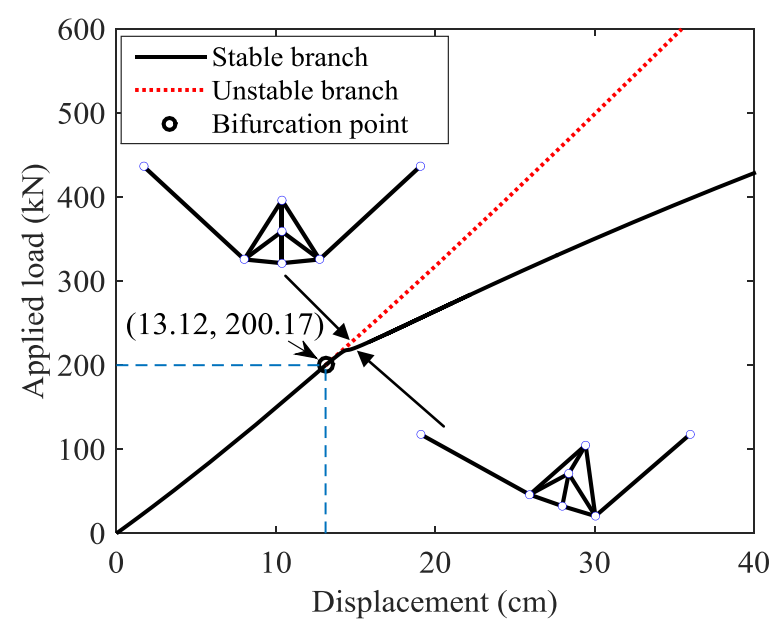

(c)

Figure 17. Load-displacement curves for optimum designs of clamped domain problem in critical load constraint formulation: (a) $P=10 \mathrm{kN}$; (b) $P=100 \mathrm{kN}$; (c) $P=200 \mathrm{kN}$.

The load-displacement behavior of optimal topologies is further analyzed by carrying out the nonlinear analysis using the arc-length and secondary path switching method described in Section 2.4. The analysis results for different topologies are shown in Figure 17. It can be observed from Figure 17 that the first critical load can be approximated as the prescribed critical load constraint factor times the applied load, i.e. $P_{c} \approx \lambda_{c r} P$. Before the first bifurcation point, the equilibrium path is stable but after the first bifurcation point, one branch becomes unstable while the other branch is still stable until the limit point is reached (Figure 17b and Figure 17c). Thus, 
1 the proposed formulation with critical load constraint is able to ensure that the topology is stable

2 for the applied load level as $P \leq P_{c} \approx \lambda_{c r} P$. In Figure 17, the deformed shapes for equilibrium

3 branches after the first critical points are also presented which show the two different

4 deformation modes after the bifurcation point is reached.

\subsubsection{Results summary}

6 The results for this problem are summarized in Table 3, in which the final compliance and the

7 first critical load are listed for each case. It can be observed that for the designs obtained from

8 compliance formulation, the critical loads are below the applied loads. With the enforcement of

9 the critical load factor constraint, however, the first critical load is always above the applied

10 loads. Although the final compliances of topologies with the critical load constraint formulation

11 (Eq. (31)) are higher than the compliance formulation (Eq. (30)), the results are more realistic as

12 stability of the final topologies is ensured in the compliance formulation with added critical load

13 factor constraint.

\begin{tabular}{cllc}
\hline Formulations & \multicolumn{1}{c}{ Cases } & $\begin{array}{c}\text { Final compliance } f_{0} \\
(\mathrm{kN} \cdot \mathrm{m})\end{array}$ & Critical load $(\mathrm{kN})$ \\
\hline & Linear elastic $P=10 \mathrm{kN}$ & 0.059 & $5.66 \times 10^{-5}$ \\
Compliance & $P=10 \mathrm{kN}$ & 0.059 & $3.96 \times 10^{0}$ \\
formulation & $P=100 \mathrm{kN}$ & 6.602 & $1.80 \times 10^{1}$ \\
& $P=200 \mathrm{kN}$ & 26.195 & $3.89 \times 10^{1}$ \\
\hline \multirow{2}{*}{$\begin{array}{c}\text { constraint } \\
\text { formulation }\end{array}$} & $P=10 \mathrm{kN}, \quad \lambda_{c r}=1.0$ & 0.060 & 10.02 \\
& $P=200 \mathrm{kN}, \quad \lambda_{c r}=1.0$ & 6.615 & 100.23 \\
\hline
\end{tabular}


1 In the second example, a tower subjected to the vertical load, as shown in Figure 18(a) is studied.

2 The domain is modeled using a ground structure with $3 \times 5$ nodes and full connectivity is assumed,

3 as shown in Figure 18(b). The available material volume for the design is limited to $V_{f}=200$

$4 \mathrm{~cm}^{3}$ and the domain dimension is $L=100 \mathrm{~cm}$. Symmetry is enforced along the vertical direction

5 according to the geometry of problem configuration.

\section{$6 \quad$ 6.2.1 Compliance formulation}

7 This problem is first solved using the compliance formulation with both the linear and nonlinear

8 truss models for $P=300 \mathrm{kN}$, and the results are shown in Figure 18(c) and Figure 18(d),

9 respectively. The results from the linear and nonlinear models are the same for this case. As there

10 is no stability constraint, all the material is located in the vertical members to minimize the

11 displacement under the load, as expected.

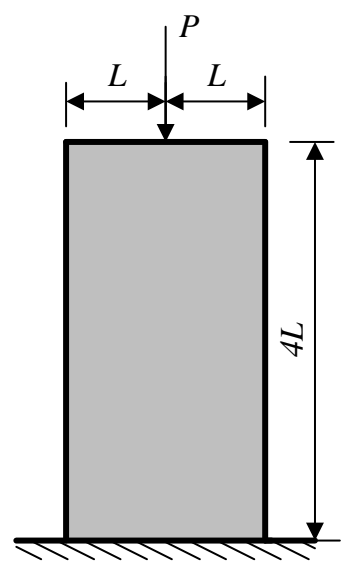

(a)

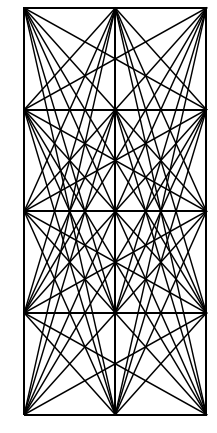

(b)

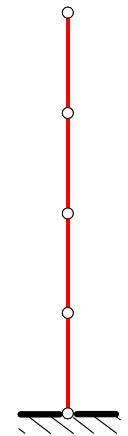

(c)

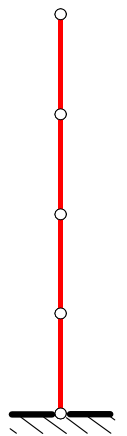

(d)

Figure 18. Tower problem: (a) Design domain; (b) Ground structure; (c) Linear result from compliance formulation; (d) Nonlinear result from compliance formulation.

The critical load analysis is carried out on the topology shown in Figure 18(d), and the first three critical load factors and corresponding eigenmodes are shown in Figure 19. The spurious modal energy ratios $\left(E S_{r}\right)$ indicate that the first three critical modes are true modes; however, these modes are associated with eigenvalues $\lambda_{r}<1.0$. Indeed, all the eigenvalues are very small 
1 indicating that the critical load is exceeded for this topology (i.e. $P>P_{c} \approx \lambda_{1} P$ ), and this topology is therefore unstable. This is further confirmed by the eigenvalue analysis of the tangent

3 stiffness matrix, which shows that the first four eigenvalues are negative as indicated in Table 4.

4 Thus, similar to the first example, with the compliance formulation the final design converged to

5 an unstable topology. Although the collinear compressive bars can be merged into a one long bar,

6 the merged single member will still be unstable.

\begin{tabular}{cccc}
\hline & Initial configuration & \multicolumn{2}{c}{ Final configuration } \\
\hline & First eigenvalue & First eigenvalue & Second eigenvalue \\
\hline \multirow{2}{*}{$P=300 \mathrm{kN}$} & $1.3335 \times 10^{-6}$ & $-1.0922 \times 10^{1}$ & $-7.2588 \times 10^{0}$ \\
\cline { 3 - 4 } & & Third eigenvalue & Fourth eigenvalue \\
\cline { 3 - 4 } & & $-3.0923 \times 10^{0}$ & $-3.7293 \times 10^{-1}$ \\
\hline
\end{tabular}

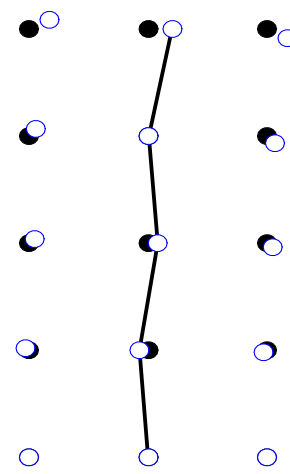

(a) $\begin{aligned} \lambda_{1} & =5.04 \times 10^{-6} \\ E S_{1} & =2.41 \times 10^{-7}\end{aligned}$

\section{formulation}

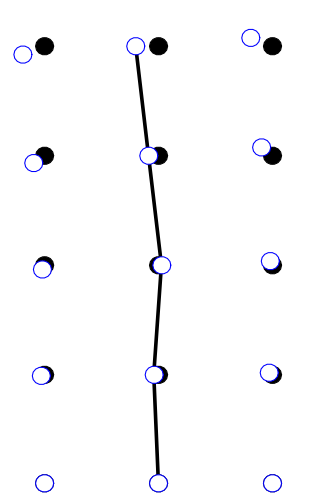
(b) $\lambda_{2}=5.63 \times 10^{-6}$
$E S_{2}=5.48 \times 10^{-7}$

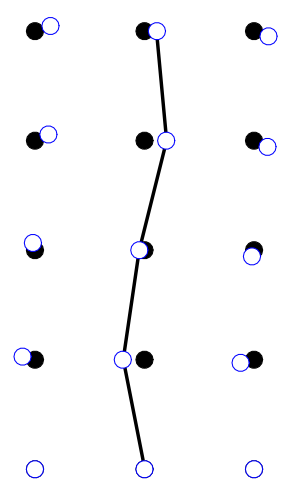

(c) $\begin{aligned} \lambda_{3} & =6.86 \times 10^{-6} \\ E S_{3} & =4.00 \times 10^{-7}\end{aligned}$

Figure 19. Critical modes of compliance formulation results for tower problem: (a) First mode; (b) Second mode; (c) Third mode.

The convergence histories of the compliance, critical load factor, and volume constraint during optimization process for the nonlinear compliance formulation are shown in Figure 20. These results again show that even if the compliance is optimized and the volume constraint is satisfied, the stability of topologies is not ensured with the compliance formulation. 


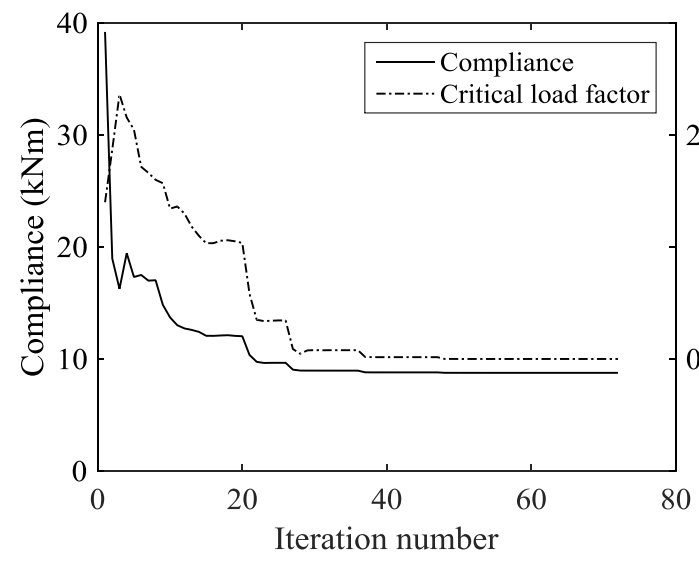

(a)

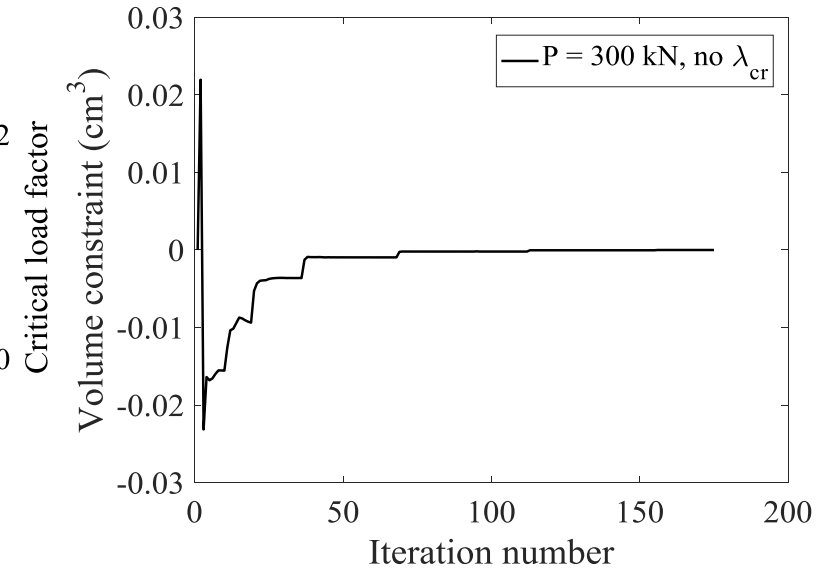

(b)

Figure 20. Iteration history curves of tower problem in compliance formulation $(P=300 \mathrm{kN})$ : (a) Compliance and critical load factor; (b) Volume constraints.

\subsubsection{Compliance formulation with critical load constraint}

This problem is again solved using the compliance formulation with critical load factor constraint of $\lambda_{c r}=1.0$. Optimization is carried out for three different load magnitudes of $P=50$ $\mathrm{kN}, P=100 \mathrm{kN}$ and $P=150 \mathrm{kN}$, and the final topology results are shown in Figure 21, where the topologies obtained from this formulation are stable as compared to the results obtained from compliance formulation (Figure 18). At a relatively small load of $P=50 \mathrm{kN}$, the vertical bar members at center have the most of material (Figure 21(a)), but braces are also present to support the collinear nodes along the length. With the increased load of $P=100 \mathrm{kN}$, the areas of the vertical bar members at the center are reduced and more material/members are present in the supporting system as shown in Figure 21(b). This feature is more pronounced at a higher load of $P=150 \mathrm{kN}$, where more members are present to support the vertical members at the center. The

first critical eigenmodes for topologies in Figure 21 are shown in Figure 22, and all the critical load factors satisfy the prescribed constraint, i.e. $\lambda_{c r}-\lambda_{1} \leq 0$ with $\lambda_{c r}=1.0$. 


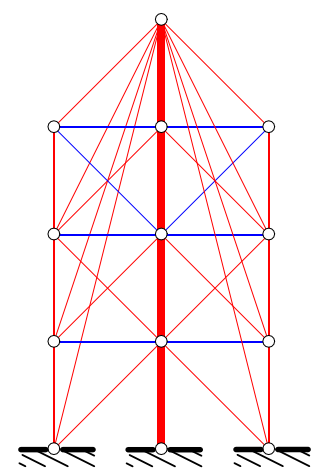

(a)

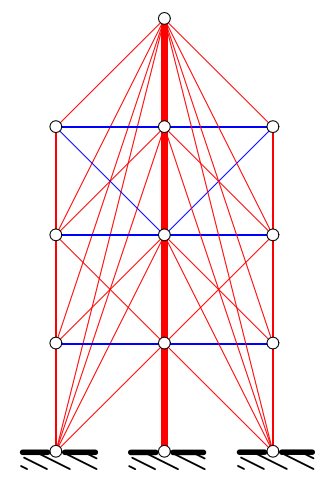

(b)

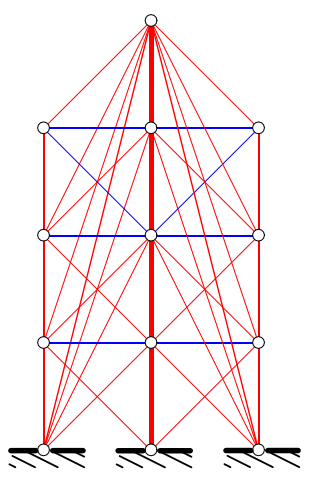

(c)

Figure 21. Tower problem with critical load constraint $\left(\lambda_{c r}=1.0\right)$ : (a) $P=50 \mathrm{kN}$; (b) $P=100$ $\mathrm{kN}$; (c) $P=150 \mathrm{kN}$.

5

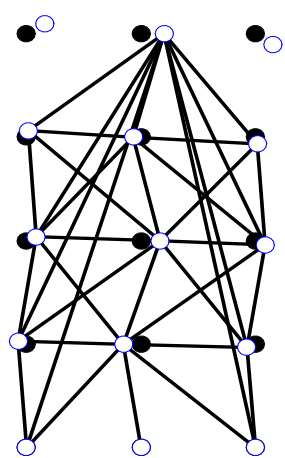

(a) $\lambda_{1}=1.00$ $E S_{1}=5.52 \times 10^{-5}$

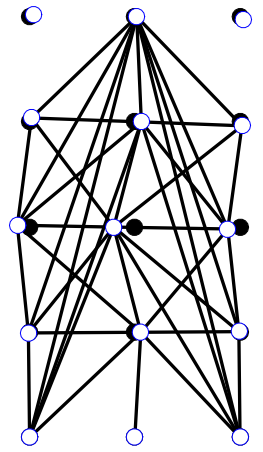

(b) $\lambda_{1}=1.00$

$E S_{1}=6.61 \times 10^{-5}$

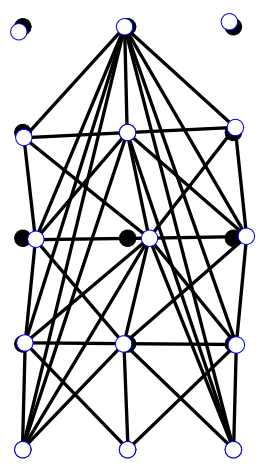

(c) $\lambda_{1}=1.00$

$E S_{1}=9.33 \times 10^{-5}$

Figure 22. First mode for tower problem $\left(\lambda_{c r}=1.0\right.$ ): (a) $P=50 \mathrm{kN}$; (b) $P=100 \mathrm{kN}$; (c) $P=150$ $\mathrm{kN}$.

The convergence histories of the compliance, critical load factor, and volume constraint during optimization process for different load cases are shown in Figure 23. As evident from these results, the convergence is achieved within 230 steps and both the critical load factor and volume constraints are adequately enforced during the optimization process. It is observed that there is a violation of the critical load factor constraint at a few steps; however, all the constraints are eventually satisfied as the optimization proceeds. 


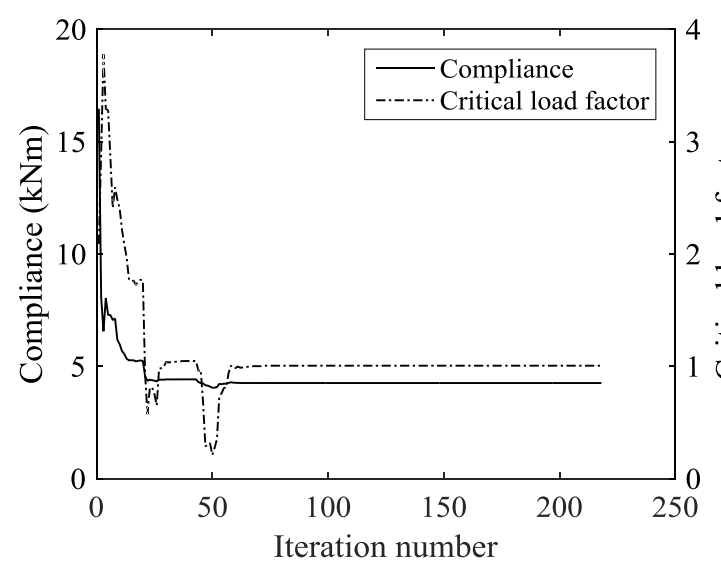

(a)

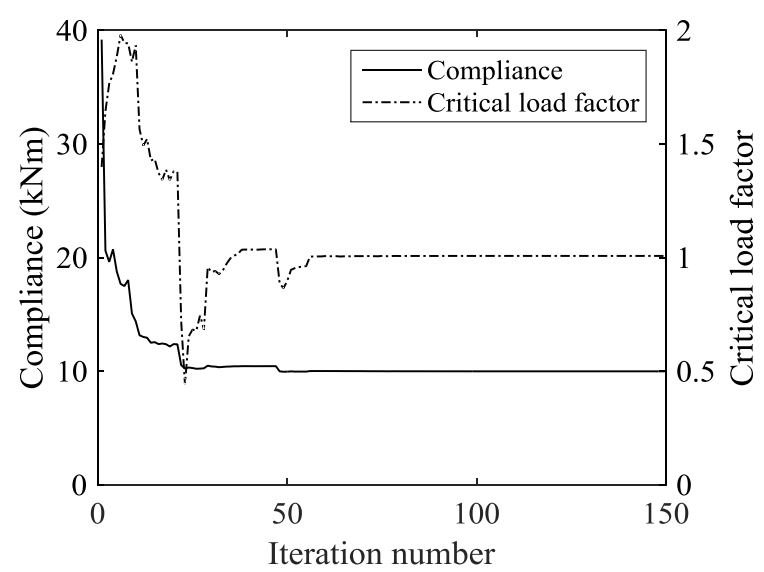

(c)

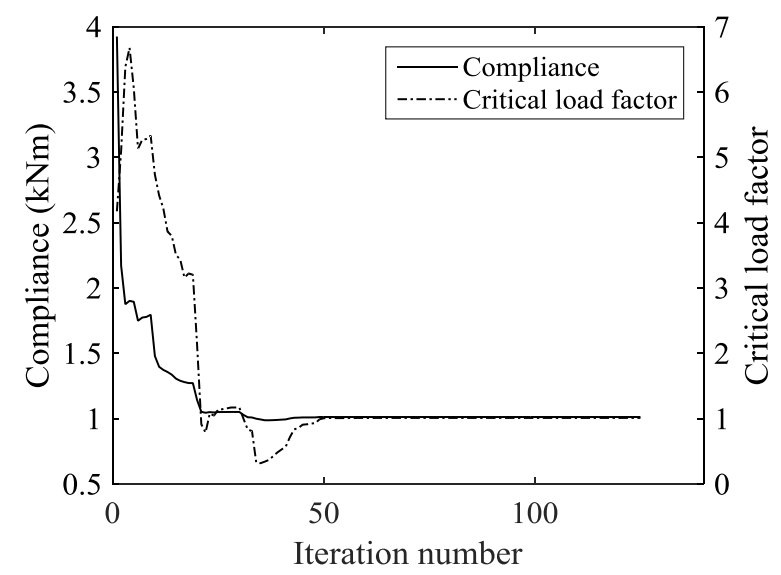

(b)

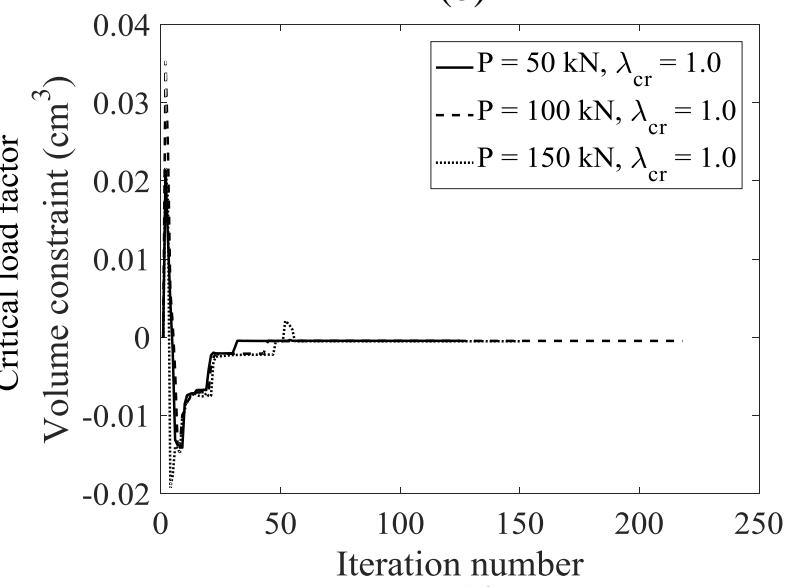

(d)

Figure 23. Convergence histories for tower problem $\left(\lambda_{c r}=1.0\right.$ ): (a) $P=50 \mathrm{kN}$; (b) $P=100 \mathrm{kN}$; (c) $P=150 \mathrm{kN}$; (d) Volume constraints.

Another parametric study is carried out on this example problem, in which the applied load is fixed to $P=50 \mathrm{kN}$ and different critical load factor constraints of $\lambda_{c r}=1.0,2.0$ and 3.0 are used. The results for this parametric study are shown in Figure 24. It is noted that for a small critical load factor constraint value (i.e. $\lambda_{c r}=1.0$ ), members with smaller areas are needed to support the vertical members at the center as shown in Figure 24(a). If the critical load factor becomes large (i.e. $\lambda_{c r}=2.0$ and 3.0), more supporting members with larger areas are introduced as shown in Figure 24(b) and Figure 24(c). Since the first critical load $\left(P_{c}\right)$ is approximately equal to $\lambda_{c r} P$, the critical load factor constraint $\lambda_{c r}$ can be considered as a factor 
1 of safety against structural instability. Therefore, when $\lambda_{c r}$ is increased the supporting members

2 become larger to enhance the stability of the system.

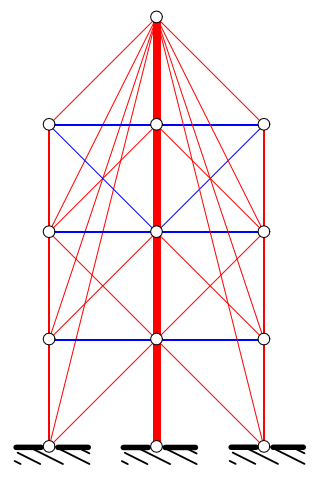

(a)

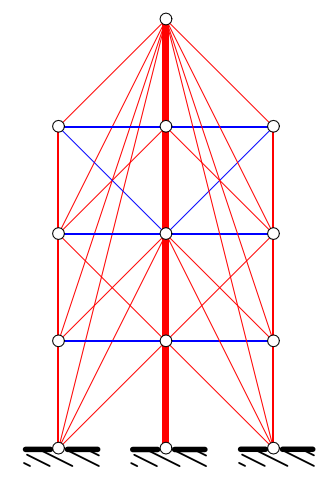

(b)

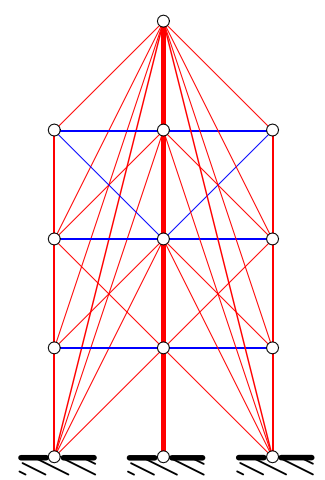

(c)
3

Figure 24. Tower problem with critical load constraint $(P=50 \mathrm{kN})$ : (a) $\lambda_{c r}=1.0$; (b) $\lambda_{c r}=2.0$; (c) $\lambda_{c r}=3.0$.

The load-displacement response curves for each case using the arc-length and secondary path switching method are presented in Figure 25. It is shown in Figure 25(a) that the initial design has a bifurcation point at $P=211.7 \mathrm{kN}$. With $\lambda_{c r}=1.0$, the optimized topologies have the bifurcation points at $P=50.64 \mathrm{kN}, 100.71 \mathrm{kN}$ and $151.13 \mathrm{kN}$ for applied loads of $P=50 \mathrm{kN}$, $100 \mathrm{kN}$ and $150 \mathrm{kN}$, as shown in Figure 25(b), Figure 25(c) and Figure 25(d) respectively, which implies that the topologies are always stable under the applied loads. Although the first critical load of the optimized topologies is lower than that of the initial design, the optimized topologies have lower compliance than that of the initial design. Moreover, additional safety can be introduced by increasing the critical load factor constraint $\left(\lambda_{c r}\right)$ at an applied load level as shown in Figure 25(e) and Figure 25(f). If $\lambda_{c r}=2.0$ and $\lambda_{c r}=3.0$ are used at a load of $P=50 \mathrm{kN}$, the first critical loads for the optimized topologies are $100.10 \mathrm{kN}$ and $149.25 \mathrm{kN}$, respectively. Thus, increasing the critical load factor constraint $\left(\lambda_{c r}\right)$ also increases the margin of safety against instability.

\subsubsection{Results summary}


1 The final results for this problem are summarized in Table 5, in which the final compliance and

2 the first critical load are listed for each case. Since the linear elastic and nonlinear cases have the

3 same designs for the compliance formulation, the first critical loads are also the same and are

4 much lower than the applied load. With the enforcement of critical load constraint, the first

5 critical loads are increased and are approximately equal to $\lambda_{c r} P$, as expected. Again, the final

6 compliances for critical load constraint formulation are higher than the compliance formulation,

7 since more material has to be distributed to members that contribute to the stability of topologies.

\begin{tabular}{clcc}
\hline \multirow{2}{*}{ Formulations } & \multicolumn{1}{c}{ Cases } & $\begin{array}{c}\text { Final compliance } f_{0} \\
(\mathrm{kN} \cdot \mathrm{m})\end{array}$ & $\begin{array}{c}\text { First critical load } \lambda_{1} P \\
(\mathrm{kN})\end{array}$ \\
\hline \multirow{2}{*}{ Compliance } & $P=50 \mathrm{kN}$ & 0.959 & $1.1 \times 10^{-3}$ \\
formulation & $P=100 \mathrm{kN}$ & 3.865 & $1.1 \times 10^{-3}$ \\
& $P=150 \mathrm{kN}$ & 8.762 & $1.1 \times 10^{-3}$ \\
\hline \multirow{2}{*}{ Critical load } & $P=50 \mathrm{kN}, \quad \lambda_{c r}=1.0$ & 1.013 & 50.71 \\
constraint & $P=150 \mathrm{kN}, \quad \lambda_{c r}=1.0$ & 4.264 & 100.71 \\
formulation & $P=50 \mathrm{kN}, \quad \lambda_{c r}=1.0$ & 9.994 & 151.13 \\
& $P=50 \mathrm{kN}, \quad \lambda_{c r}=3.0$ & 1.056 & 100.10 \\
& & 1.088 & 149.25 \\
\hline
\end{tabular}




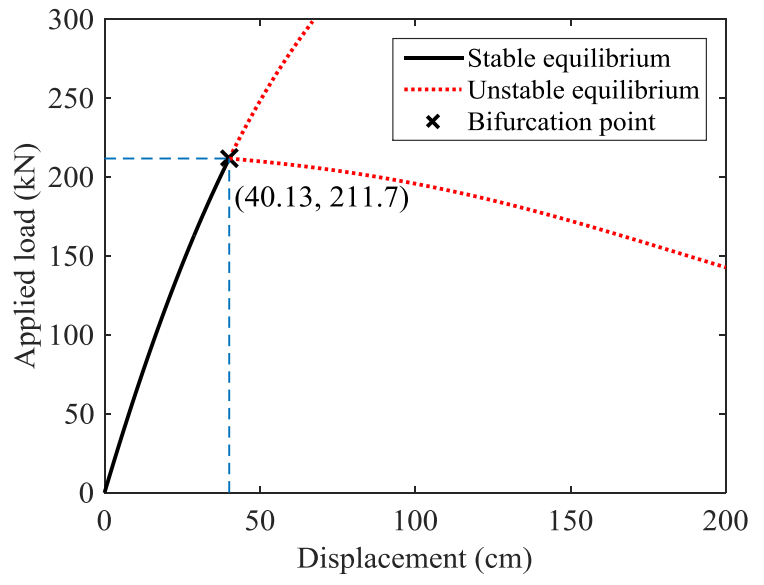

(a)

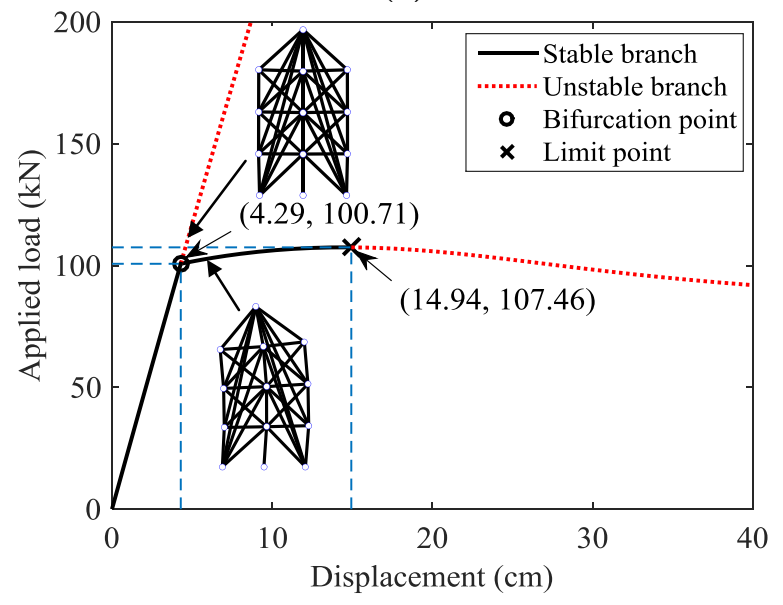

(c)

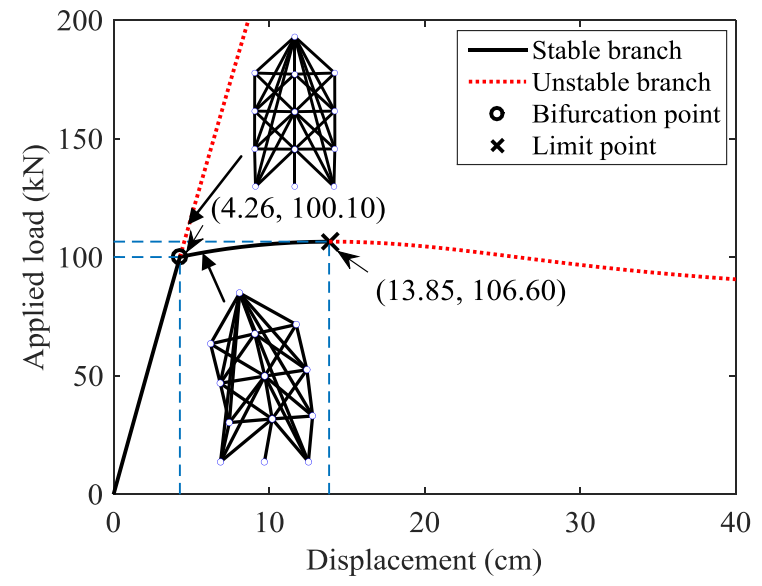

(e)

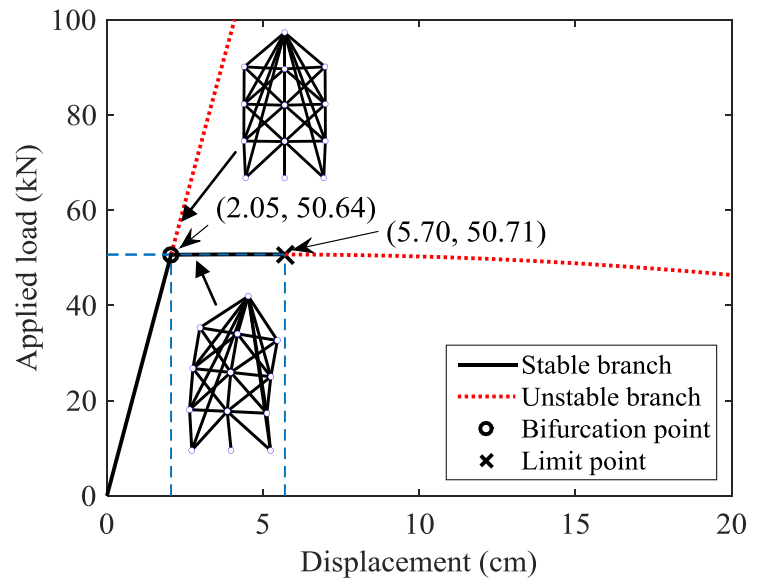

(b)

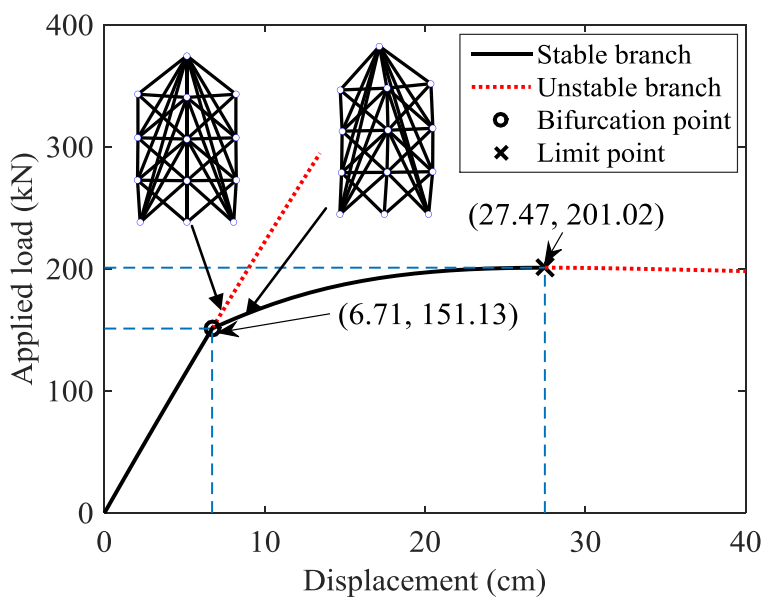

(d)

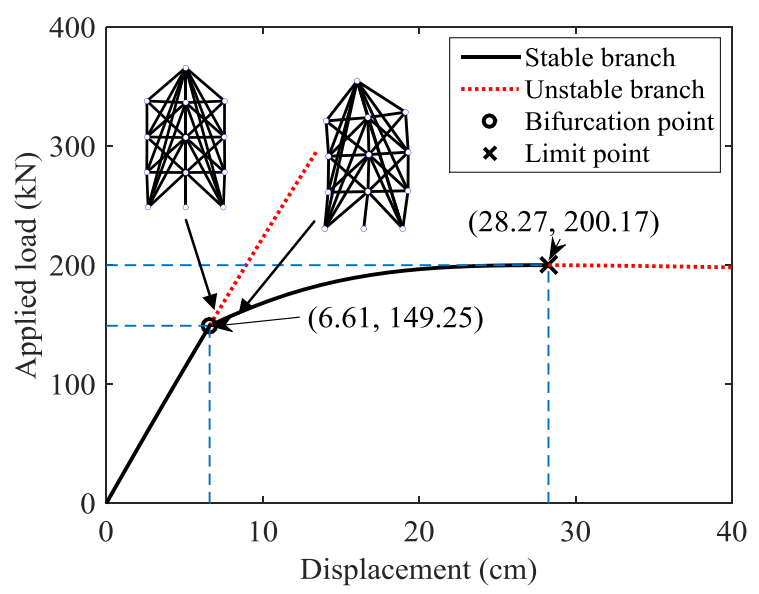

(f)
1

Figure 25. Load-displacement curves for tower problem: (a) Initial design; (b) $P=50 \mathrm{kN}, \lambda_{c r}=$ 1.0 ; (c) $P=100 \mathrm{kN}, \lambda_{c r}=1.0$; (d) $P=150 \mathrm{kN}, \lambda_{c r}=1.0$; (e) $P=50 \mathrm{kN}, \lambda_{c r}=2.0$; (f) $P=50 \mathrm{kN}$, $\lambda_{c r}=3.0$. 


\subsection{Asymmetrically loaded tower problem}

2 The last example consists of an asymmetrically loaded tower as shown in Figure 26(a) and the

3 initial ground structure has $4 \times 7$ nodes with full connectivity as shown in Figure 26(b). The

4 available material volume for the design is limited to $V_{f}=300 \mathrm{~cm}^{3}$ and the domain dimension is

$5 \quad L=100 \mathrm{~cm}$.

\section{$6 \quad$ 6.3.1 Compliance formulation}

7 Unlike the previous two examples, which have the symmetric loading and geometry

8 configurations, this problem is asymmetrically loaded. This ground structure first is optimized

9 for an applied load of $P=50 \mathrm{kN}$ using the nonlinear truss model. However, during the 10 optimization steps, the topologies in this example problem have limit load of less than $50 \mathrm{kN}$, 11 and the load control based NR solution process fails in this case. For instance, the structural 12 response of the topology at the optimization iteration 103, obtained using the arc-length method, 13 is shown in Figure 27. At this step, the limit load for the topology is around $26.6 \mathrm{kN}$ and there is 14 no convergence in the load control based NR solution process. In essence, the sensitivity 15 analyses cannot be carried out at this step and the optimization process cannot proceed any 16 further. Thus, this ground structure cannot be optimized using the compliance formulation with 17 nonlinear truss model. Therefore, this problem is first solved using the linear truss model with $P=50 \mathrm{kN}$ and the result is shown in Figure 28(a). It is observed that the collinear bars in

19 compression exist in this topology and this topology is clearly unstable. The topology shown in 20 Figure 28(a) is analyzed using the nonlinear model with $P=50 \mathrm{kN}$ and the first three critical 21 modes are shown in Figure 28(b)-(d). The spurious modal energy ratios $\left(E S_{r}\right)$ indicate that the 22 first three critical modes are true modes; however, these modes are associated with eigenvalues $23 \lambda_{k}<1.0$ and this topology is unstable as $P>P_{c} \approx \lambda_{1} P$. 


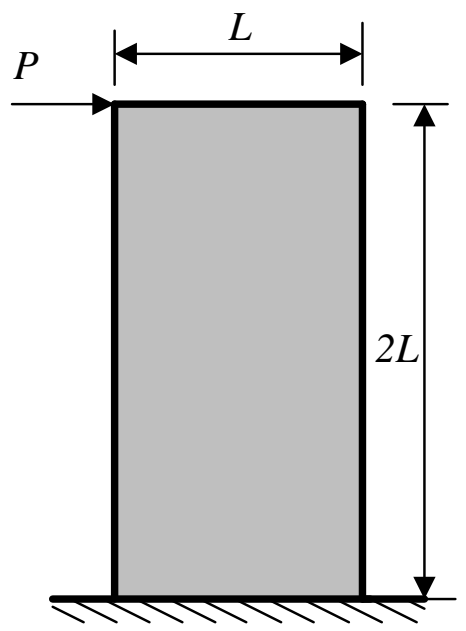

(a)

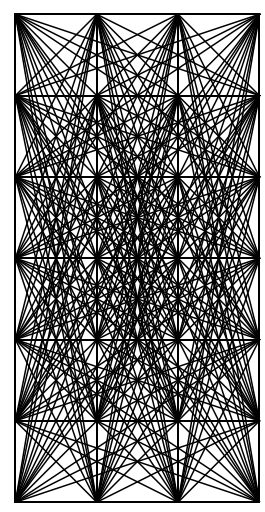

(b)

Figure 26. Asymmetrically loaded tower problem: (a) Design domain; (b) Ground structure.

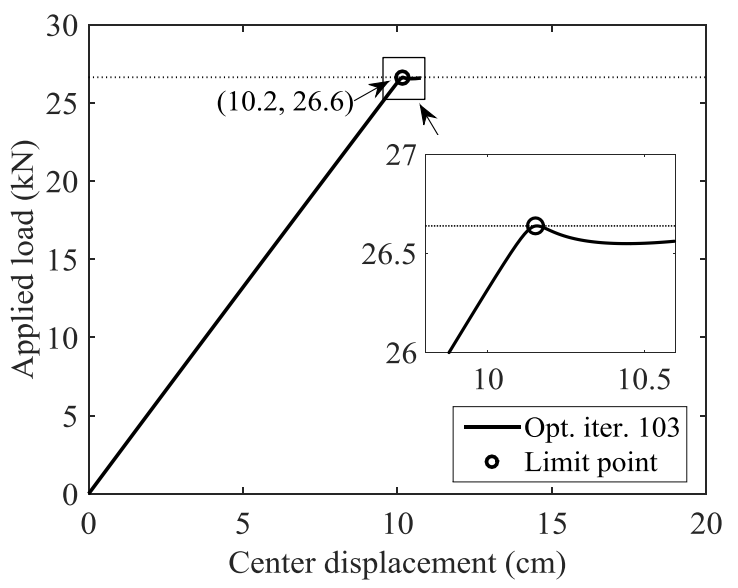

Figure 27. Load-displacement response of topology at optimization iteration \#103.

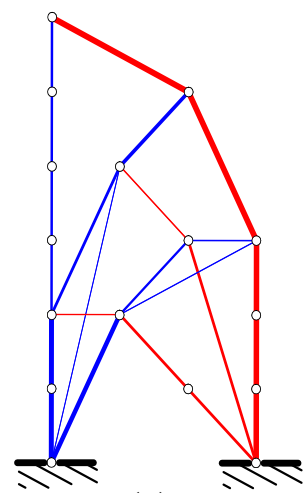

(a)

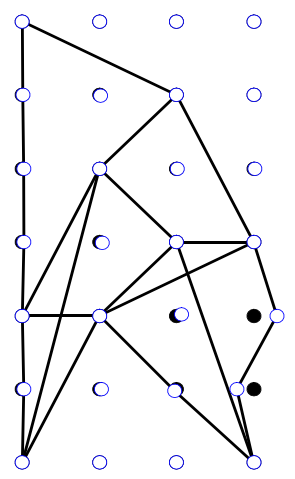

(b) $\lambda_{1}=5.81 \times 10^{-7}$ $E S_{1}=9.21 \times 10^{-10}$

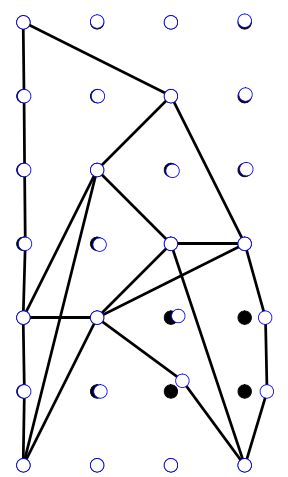

(c) $\lambda_{2}=1.42 \times 10^{-6}$ $E S_{2}=1.79 \times 10^{-9}$

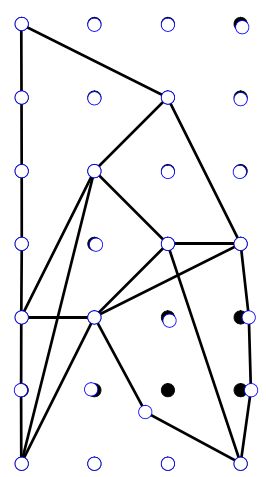

(d) $\lambda_{3}=3.49 \times 10^{-6}$ $E S_{3}=2.54 \times 10^{-9}$

Figure 28. Critical point modes for linear result of asymmetrically loaded tower: (a) Optimal design; (b) First mode; (c) Second mode; (d) Third mode. 
2 This problem is again solved with $P=50 \mathrm{kN}$, and to avoid convergence issues during the NR

3 solution process a critical load constraint value of $\lambda_{c r}>1.0$ is used, as the critical point in this

4 case is a limit point. Three values of $\lambda_{c r}=1.2,2.0$ and 3.0 are used and the final topology

5 results are shown in Figure 29(a), Figure 29(b) and Figure 29(c), respectively. These results

6 indicate that more members are introduced into the final topologies to support the collinear bars.

7 The additional members are mostly distributed around the collinear bars in the compression zone

8 to enhance the stability and more braces are introduced with increase in the critical load factor

9 constraint $\lambda_{c r}$. An important aspect of the new formulation (Eq. (31)) is that the NR method 10 always converges, which is not the case for the compliance formulation (Eq. (30)). This feature 11 is desirable for the presented nonlinear asymmetric topology optimization problem.

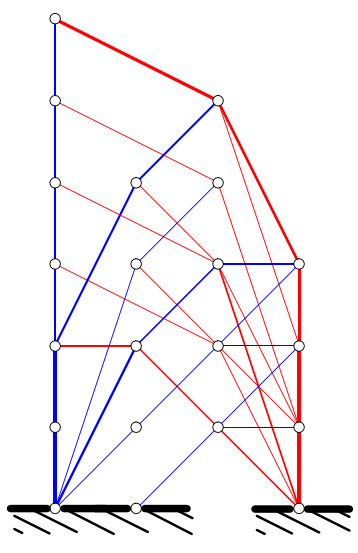

(a)

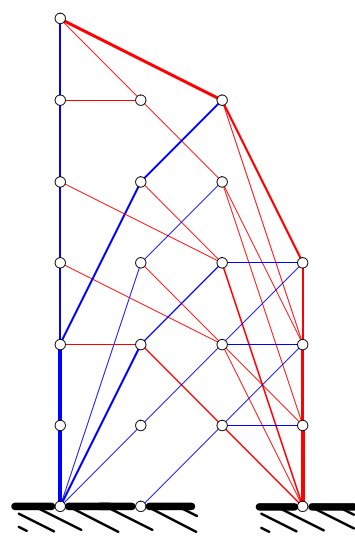

(b)

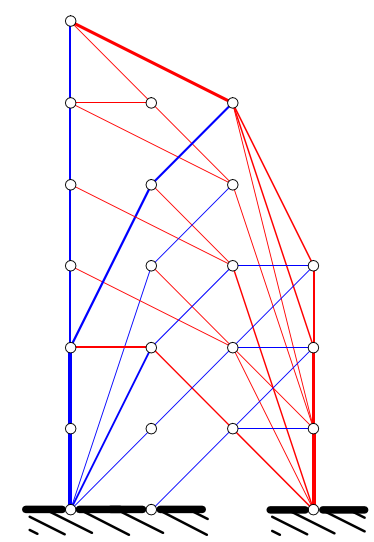

(c)

Figure 29. Final topologies of asymmetrically loaded tower in critical load constraint formulation $(P=50 \mathrm{kN})$ : (a) $\lambda_{c r}=1.2$; (b) $\lambda_{c r}=2.0$; (c) $\lambda_{c r}=3.0$.

The first three eigenmodes of the optimized topologies are shown in Table 6. It can be seen that the critical load factor constraint is enforced for each case (i.e. $\lambda_{c r}-\lambda_{1} \leq 0$ ) and the first three eigenvalues are different implying that the eigenvalues are simple. The spurious modal energy ratios $\left(E S_{k}\right)$ indicate that all these modes are true modes. These modes indicate that the majority 
1 of deformation still occurs in the compression zone. However, the stability of compression zone

2 is enhanced during the optimization process to achieve the desired critical load.

Cases

Table 6. First three modes for different $\lambda_{c r}$

4 The convergence histories of the compliance, critical load factor, and volume constraint during

5 optimization process are shown in Figure 30. These plots indicate that the objective function

6 decreases as the optimization proceeds and the volume constraint is adequately enforced. The

7 critical load factor constraint is violated at few steps during the optimization process, but finally

8 converges to the prescribed values of $\lambda_{c r}=1.2,2.0$ and 3.0, respectively. 


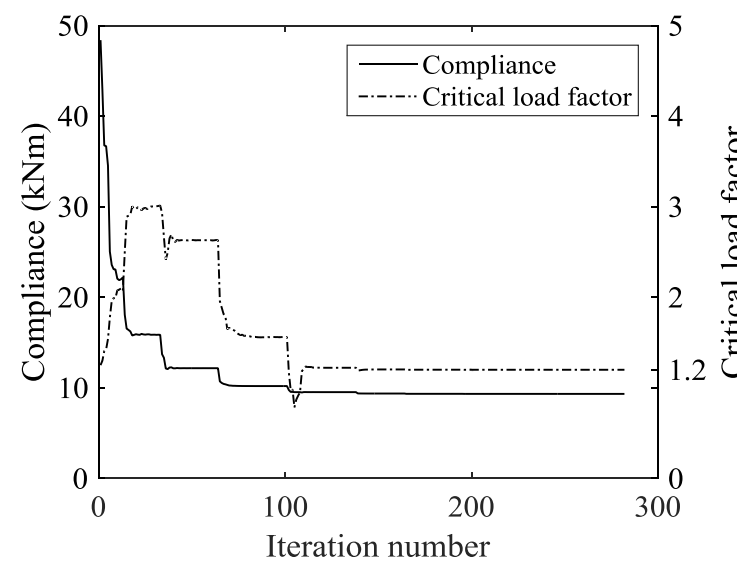

(a)

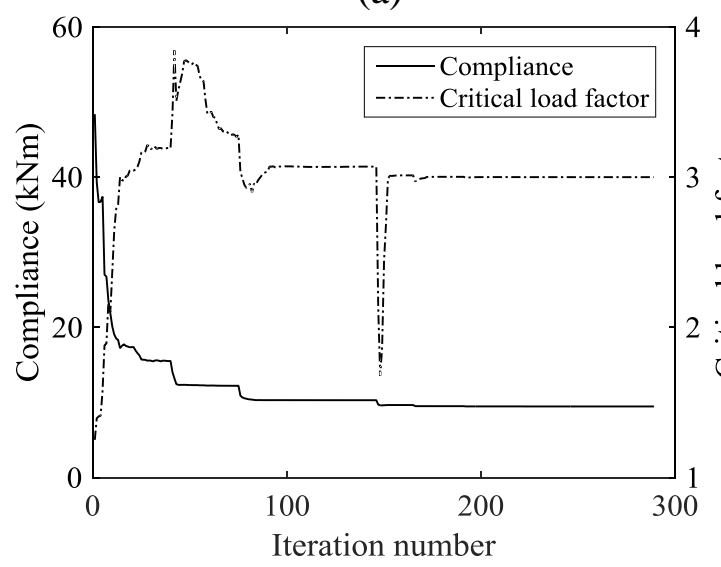

(c)

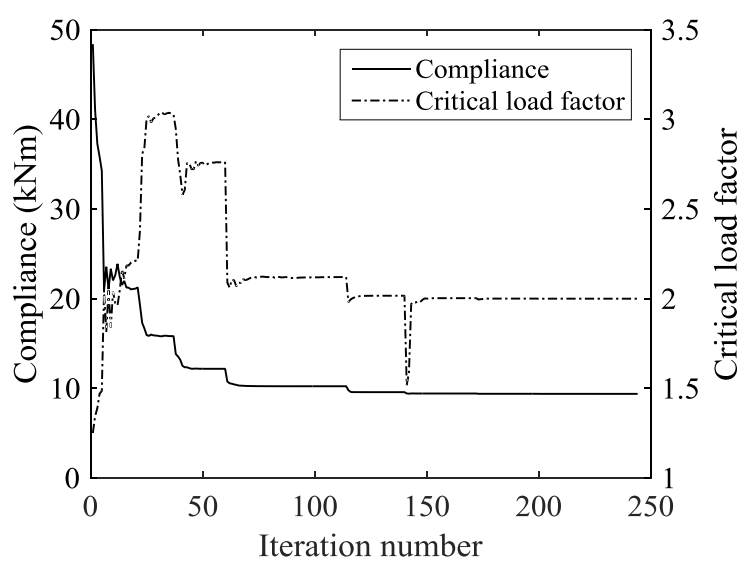

(b)

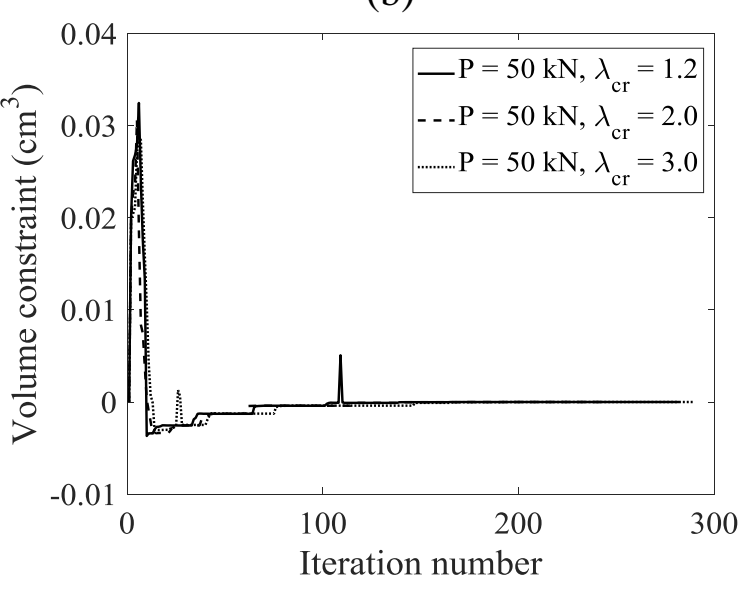

(d)

Figure 30. Convergence histories for asymmetrically loaded tower $(P=50 \mathrm{kN})$ : (a) $\lambda_{c r}=1.2$; (b) $\lambda_{c r}=2.0 ;$ (c) $\lambda_{c r}=3.0 ;$ (d) Volume constraints.

4 An additional parametric study is carried out for this example, and a larger applied load of $P=$

$5100 \mathrm{kN}$ is used with critical load constraints of $\lambda_{c r}=1.2,1.5$ and 2.0 and the results are shown

6 in Figure 31. It is observed that with the increase in load, similar topologies are obtained.

7 However, more material is present in the key load carrying members as compared to the results

8 shown in Figure 29 for $P=50 \mathrm{kN}$.

9 In Figure 32, the load-displacement response curves are presented for the final topology designs

10 of the nonlinear asymmetrically loaded tower problem. The results of the load case $P=50 \mathrm{kN}$

11 are shown in Figure 32(a) while results of the load case $P=100 \mathrm{kN}$ are shown in Figure $32(\mathrm{~b})$,

12 together with response of the initial design. It is evident from the results that the stiffness is 
1 increased after optimization as compared to the initial design. Moreover, different critical load

2 factor constraints introduce different critical points. In this example, all the critical points are

3 limit points and the critical load $\left(P_{c}\right)$ at limit points is close to $\lambda_{c r} P$, as expected. Furthermore,

4 the equilibrium path is stable under the applied loads for all the cases.

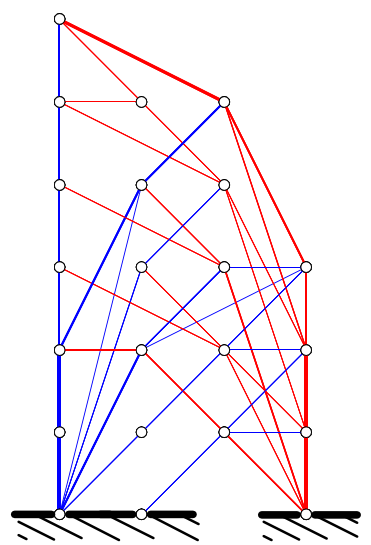

(a)

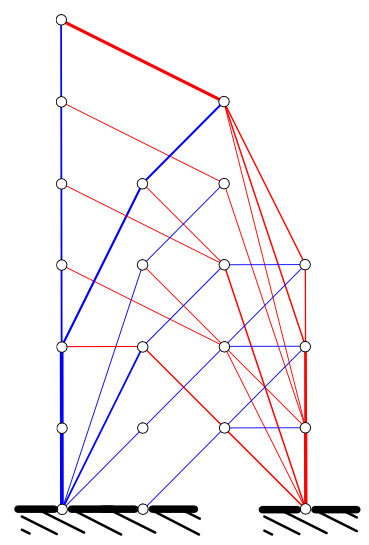

(b)

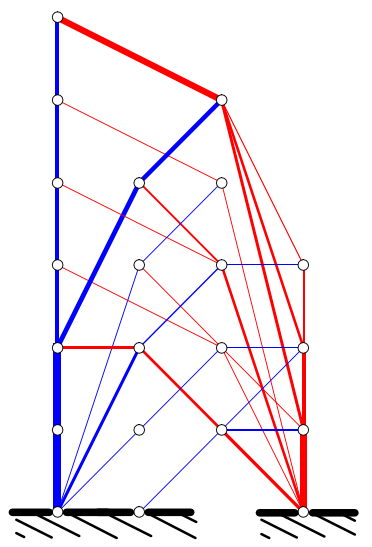

(c)

$6 \quad$ Figure 31. Final topologies of asymmetrically loaded tower problem in critical load constraint formulation $(P=100 \mathrm{kN})$ : (a) $\lambda_{c r}=1.2$; (b) $\lambda_{c r}=1.5$; (c) $\lambda_{c r}=2.0$.

\subsubsection{Results summary}

The results for the asymmetrically loaded tower problem are summarized in Table 7, in which the final compliance and the first critical load are listed for each case. Since only the linear elastic model works for compliance formulation, the nonlinear analyses of linear elastic design under loads of $P=50 \mathrm{kN}$ and $P=100 \mathrm{kN}$ are carried out. The first critical loads for the linear design are the same and are much lower than the applied loads. The first critical loads are increased approximately to $P_{c} \approx \lambda_{c r} P$ with the enforcement of the critical load factor constraint, ensuring the stability of the topologies. Moreover, the final compliances for nonlinear trusses with critical load constraint are lower than those for linear trusses without any stability constraint. 


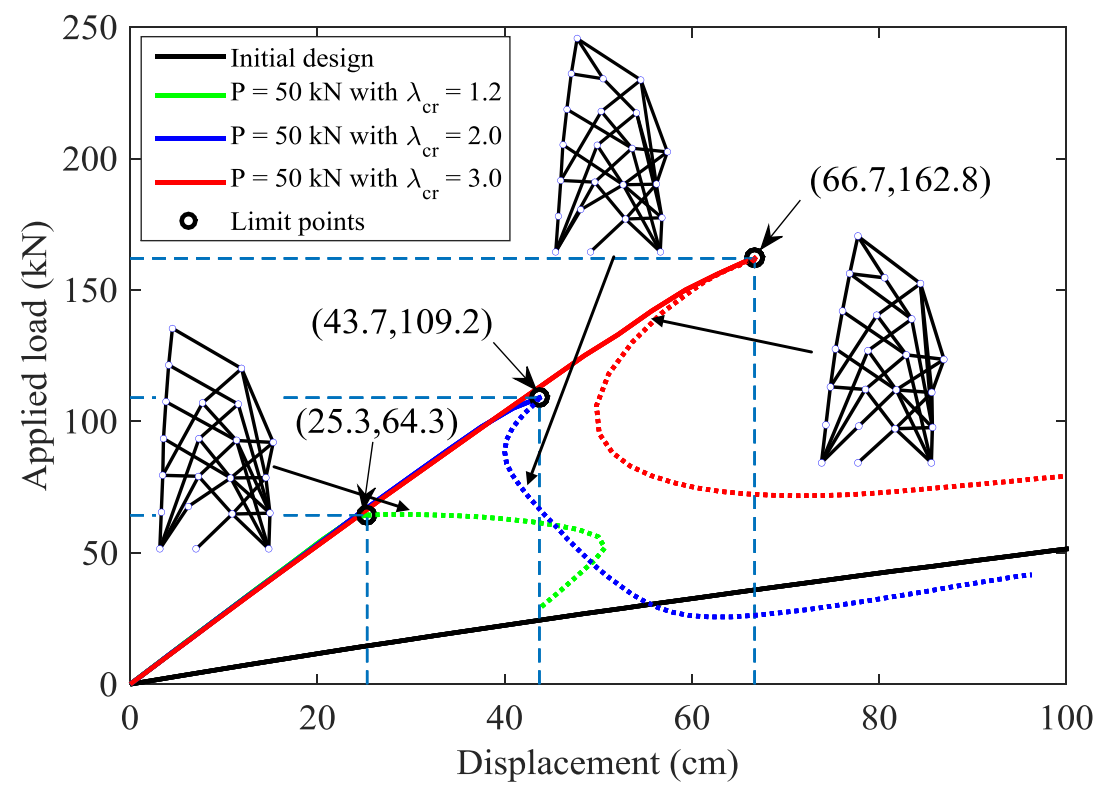

(a)

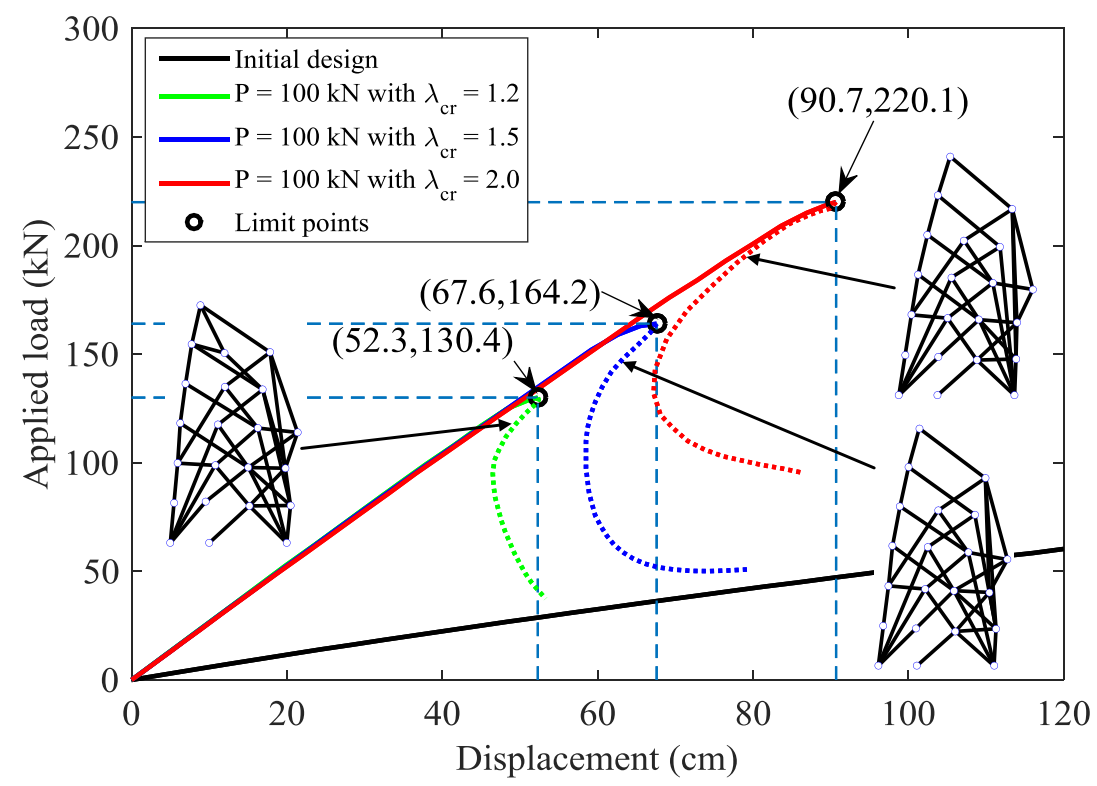

(b)

Figure 32. Load-displacement curves for asymmetrically loaded tower: (a) $P=50 \mathrm{kN}$; (b) $P=$ 3 $100 \mathrm{kN}$. 


\begin{tabular}{cccc}
\hline Formulations & Cases & $\begin{array}{c}\text { Final compliance } f_{0} \\
(\mathrm{kN} \cdot \mathrm{m})\end{array}$ & $\begin{array}{c}\text { First critical load } \lambda_{1} P \\
(\mathrm{kN})\end{array}$ \\
\hline $\begin{array}{c}\text { Compliance } \\
\text { formulation }\end{array}$ & $\begin{array}{c}\text { Linear elastic } P=50 \mathrm{kN} \\
\text { Linear elastic } P=100 \mathrm{kN}\end{array}$ & $\begin{array}{c}14.135 \\
58.685\end{array}$ & $\begin{array}{c}2.61 \times 10^{-5} \\
2.61 \times 10^{-5}\end{array}$ \\
\hline & $P=50 \mathrm{kN}, \quad \lambda_{c r}=1.2$ & 9.338 & 64.33 \\
Critical load & $P=50 \mathrm{kN}, \quad \lambda_{c r}=3.0$ & 9.387 & 109.22 \\
formulation & $P=100 \mathrm{kN}, \quad \lambda_{c r}=1.2$ & 38.463 & 162.81 \\
& $P=100 \mathrm{kN}, \quad \lambda_{c r}=1.5$ & 38.313 & 130.43 \\
& $P=100 \mathrm{kN}, \quad \lambda_{c r}=2.0$ & 38.584 & 220.12 \\
\hline
\end{tabular}

\section{Conclusions}

3 Topology optimization of geometrically nonlinear trusses is addressed in this study. It is

4 observed that if minimum compliance formulation is used without any stability constraints, the

5 final topology designs may be unstable. Moreover, the optimization process may fail for cases

6 wherein the limit load is exceed due to the non-convergence in the NR solution process. To

7 address these issues, a minimum compliance formulation for nonlinear trusses with critical load

8 factor constraint is introduced. The constraint is based on the minimum critical load factor

9 associated with the true mode, and to accurately estimate the critical load factor the critical load

10 analysis is carried out at the last load step. A strategy based on the spurious modal energy ratio is

11 introduced to determine the spurious modes that are associated with the deformation in the low-

12 density members.

13 Topology results obtained using the critical load factor constraint show that the optimized

14 topologies are stable and there are no convergence issues in the NR process. The critical load

15 constraint ensures that the first critical load is above the applied load, and therefore stability of 
1 the equilibrium before and at the applied load level is enforced. The critical load factor $\left(\lambda_{c r}\right)$

2 used in the constraint can be also considered as a safety margin against global buckling and the

3 margin of safety can be increased by increasing the critical load constraint factor. In the load-

4 displacement response curves of the final topologies, both the bifurcation and limit points are

5 encountered. However, with the proposed formulation the load control NR solver can still be

6 used, as the first critical point is always above the applied loads.

7 In this study, only the simple eigenvalues are considered and further work in this direction may

8 include problems wherein eigenvalues have multiplicities greater than one. In particular, the

9 sensitivity analysis would have to be modified in this case. In addition, the post-critical behavior

10 is not considered in topology design in this study. As one of the solution branches after

11 bifurcation is stable with increasing loads for some cases, it may be possible to make use of this

12 additional capacity. Other important issues such as constructability and local instability need

13 further attention, and these important issues will be addressed in our future work.

\section{Acknowledgements}

15 The presented work is supported in part by the US National Science Foundation through Grant

16 CMS-1055314. Any opinions, findings, conclusions, and recommendations expressed in this

17 paper are those of the authors and do not necessarily reflect the views of the sponsors. 
[1] Bendsøe MP, Sigmund O. Topology optimization - theory, methods and applications. 2nd edn ed. Berlin: Springer; 2003.

[2] Toğan V, Daloğlu AT. Optimization of $3 \mathrm{~d}$ trusses with adaptive approach in genetic algorithms. Engineering Structures. 2006;28:1019-27.

[3] Rahami H, Kaveh A, Gholipour Y. Sizing, geometry and topology optimization of trusses via force method and genetic algorithm. Engineering Structures. 2008;30:2360-9.

[4] Souza RRd, Fadel Miguel LF, Lopez RH, Miguel LFF, Torii AJ. A procedure for the size, shape and topology optimization of transmission line tower structures. Engineering Structures. 2016;111:162-84.

[5] Kaveh A, Hassani B, Shojaee S, Tavakkoli SM. Structural topology optimization using ant colony methodology. Engineering Structures. 2008;30:2559-65.

[6] Christensen PW, Klarbring A. An introduction to structural optimization: Springer Science \& Business Media; 2008.

[7] Li L, Khandelwal K. An adaptive quadratic approximation for structural and topology optimization. Computers \& structures. 2015;151:130-47.

[8] Dorn WS, Gomory RE, Greenberg HJ. Automatic design of optimal structures. Journal de Mecanique. 1964;3:25-52.

[9] Hemp WS. Optimum structures: Clarendon Press; 1973.

[10] Achtziger W, Bendsøe M, Ben-Tal A, Zowe J. Equivalent displacement based formulations for maximum strength truss topology design. IMPACT of Computing in Science and Engineering. 1992;4:315-45.

[11] Ben-Tal A, Bendsøe MP. A new method for optimal truss topology design. SIAM journal on optimization. 1993;3:322-58.

[12] Bendsøe MP, Ben-Tal A, Zowe J. Optimization methods for truss geometry and topology design. Structural Optimization. 1994;7:141-59.

[13] Li L, Khandelwal K. Two-point gradient-based MMA (TGMMA) algorithm for topology optimization. Computers \& Structures. 2014;131:34-45.

[14] Sokół T. A 99 line code for discretized Michell truss optimization written in Mathematica. Structural and Multidisciplinary Optimization. 2011;43:181-90.

[15] Achtziger W. On simultaneous optimization of truss geometry and topology. Structural and Multidisciplinary Optimization. 2007;33:285-304.

[16] Gilbert M, Tyas A. Layout optimization of large-scale pin-jointed frames. Engineering Computations. 2003;20:1044-64.

[17] Ben-Tal A, Jarre F, Kočvara M, Nemirovski A, Zowe J. Optimal design of trusses under a nonconvex global buckling constraint. Optimization and Engineering. 2000;1:189-213.

[18] Rozvany GI. Difficulties in truss topology optimization with stress, local buckling and system stability constraints. Structural Optimization. 1996;11:213-7.

[19] Zhou M. Difficulties in truss topology optimization with stress and local buckling constraints. Structural optimization. 1996;11:134-6.

[20] Achtziger W. Local stability of trusses in the context of topology optimization part I: exact modelling. Structural optimization. 1999;17:235-46.

[21] Kočvara M. On the modelling and solving of the truss design problem with global stability constraints. Structural and Multidisciplinary Optimization. 2002;23:189-203. 
[22] Guo X, Cheng G, Olhoff N. Optimum design of truss topology under buckling constraints. Structural and Multidisciplinary Optimization. 2005;30:169-80.

[23] Lindgaard E, Lund E. Nonlinear buckling optimization of composite structures. Computer methods in applied mechanics and engineering. 2010;199:2319-30.

[24] Klarbring A, Stromberg N. Topology optimization of hyperelastic bodies including nonzero prescribed displacements. Structural and Multidisciplinary Optimization. 2013;47:37-48.

[25] Lindgaard E, Dahl J. On compliance and buckling objective functions in topology optimization of snap-through problems. Structural and Multidisciplinary Optimization. 2013;47:409-21.

[26] Khot N. Nonlinear analysis of optimized structure with constraints on system stability. AIAA journal. 1983;21:1181-6.

[27] Kamat M, Khott N, Venkayyat V. Optimization of shallow trusses against limit point instability. AIAA journal. 1984;22:403-8.

[28] Bochenek B. Optimization of geometrically nonlinear structures with respect to both buckling and postbuckling constraints. Engineering Optimization. 1997;29:401-15.

[29] Suleman A, Sedaghati R. Benchmark case studies in optimization of geometrically nonlinear structures. Structural and Multidisciplinary Optimization. 2005;30:273-96.

[30] Ramos Jr AS, Paulino GH. Convex topology optimization for hyperelastic trusses based on the ground-structure approach. Structural and Multidisciplinary Optimization. 2013;51:287-304.

[31] Neves M, Rodrigues H, Guedes J. Generalized topology design of structures with a buckling load criterion. Structural optimization. 1995;10:71-8.

[32] Pedersen NL. Maximization of eigenvalues using topology optimization. Structural and Multidisciplinary Optimization. 2000;20:2-11.

[33] Gao X, Ma H. Topology optimization of continuum structures under buckling constraints. Computers \& structures. 2015;157:142-52.

[34] Bonet J, Wood RD. Nonlinear continuum mechanics for finite element analysis: Cambridge university press; 1997.

[35] Kiran R, Li L, Khandelwal K. Performance of cubic convergent methods for implementing nonlinear constitutive models. Computers \& Structures. 2015;156:83-100.

[36] Wagner W, Wriggers P. A simple method for the calculation of postcritical branches. Engineering Computations. 1988;5:103-9.

[37] de Borst R, Crisfield MA, Remmers JJ, Verhoosel CV. Nonlinear finite element analysis of solids and structures: John Wiley \& Sons; 2012.

[38] de Souza Neto EA, Peric D, Owen DRJ. Computational methods for plasticity: theory and applications: John Wiley \& Sons; 2011.

[39] de Souza Neto EA, Feng YT. On the determination of the path direction for arc-length methods in the presence of bifurcations and 'snap-backs'. Computer methods in applied mechanics and engineering. 1999;179:81-9.

[40] Wriggers P. Nonlinear finite element methods: Springer Science \& Business Media; 2008.

[41] Lindgaard E, Lund E. Optimization formulations for the maximum nonlinear buckling load of composite structures. Structural and Multidisciplinary Optimization. 2011;43:631-46.

[42] Bathe K-J. Finite element procedures: Prentice Hall; 1996.

[43] Michaleris P, Tortorelli DA, Vidal CA. Tangent operators and design sensitivity formulations for transient non - linear coupled problems with applications to elastoplasticity. International Journal for Numerical Methods in Engineering. 1994;37:2471-99. 
1 [44] Strang G. Computational science and engineering: Wellesley-Cambridge Press Wellesley; 22007.

3 [45] Li L, Khandelwal K. Volume preserving projection filters and continuation methods in 4 topology optimization. Engineering Structures. 2015;85:144-61.

5 [46] Bertsekas DP. Nonlinear programming. 2nd ed. Belmont, Massachusetts: Athena Scientific; 61999. 